\title{
IMPROVEMENT OF YIELD AND QUALITY OF SUGAR BEET SOWN AT TWO DATES BY METHANOL AND BORON APPLICATION
}

\author{
B.S.I. Makhlouf ${ }^{(1)}$ and Soha R. Khalil (2) \\ (1) Agron., Res. Dept., ${ }^{(2)}$ Sugar Technol., Res., Dept. \\ Sugar Crops Res. Inst., Agric. Res. Center, Giza, Egypt
}

Received: Apr. 20, 2017

Accepted: Apr. 29, 2017

\begin{abstract}
Two field experiments were carried out in Senouris, Fayoum Governorate, Egypt (latitude of $30.82^{\circ} \mathrm{N}$ and longitude of $29.40^{\circ} \mathrm{E}$ ) in 2014/2015 and 2015/2016 seasons to find out the optimal sowing date, methanol and boron fertilization levels to get the highest yield and quality of sugar beet. This work included two sowing dates $\left(15^{\text {th }}\right.$ September and $15^{\text {th }}$ October), three foliar concentrations of methanol (0, 10 and $20 \%)$ and three foliar concentrations of boron (0, 0.5 and $1.0 \mathrm{~g}$ boric acid "17\%B"/l). At each sowing date, the nine combinations of methanol and boron levels were randomly distributed in a randomized complete block design with three replications. Thereafter, a combined analysis between the two sowing dates was done. Sugar beet Sara multi-germ variety was sown in both seasons.

The results revealed that sugar beet sown earlier on the $15^{\text {th }}$ of September over-passed that planted on 15 October in root length, diameter and fresh weight/plant, leaf area index (LAI), net assimilation rate (NAR), photosynthetic pigments, polyphenol, sucrose\%, extractable sugar\% (ES), purity\% and top, root and sugar yields/fed, while Na, K, $\alpha$-amino nitrogen, fiber and sugar lost to molasses\% (SLM) were decreased.

Spraying methanol at $20 \%$ and/or boron at $1.0 \mathrm{~g}$ boric acid/l led to significant increments in root length, diameter and fresh weight/plant, LAI, NAR, photosynthetic pigments, polyphenol\%, sucrose $\%$, ES\%, purity\% as well as top, root and sugar yields/fed, while $\mathrm{Na}, \mathrm{K}$ and $\alpha$-amino $\mathrm{N}$ contents, fiber\% and SLM\% were significantly decreased in both seasons.

The combination between sowing on 15 September and raising concentration of the sprayed methanol solution to 10 and $20 \%$ attained the highest root length, LAI, NAR, chlorophyll "a", carotenoids and yields of top and root compared to sowing on 15 October in both seasons, as well as root fresh weight/plant and chlorophyll "b" in the $1^{\text {st }}$ season only, and sucrose\%, ES\% and sugar yield/fed in the $2^{\text {nd }}$ one.

The interaction between sowing dates and boron significantly affected SLM\%, purity\%, Na content and root yield, in the $1^{\text {st }}$ season, as well as root length and fresh weight/plant, chlorophyll a, fiber\%, a-amino $N$ and top yield/fed, in the $2^{\text {nd }}$ one. Purity\%, LAl, NAR and polyphenol were significantly influenced by the interaction between methanol and boron levels in the $1^{\text {st }}$ season, as well as chlorophyll "b", Na and K contents, in both seasons.

Based upon the obtained results, sowing sugar beet earlier on the $15^{\text {th }}$ of September, sprayed with $20 \%$ methanol and $1.0 \mathrm{~g}$ boric acid/l can be recommended to attain the highest root and sugar yields/fed as well as the best juice quality characteristics under conditions of the present work.
\end{abstract}

Key words: Sugar beet, sowing date, methanol, boron, quality, yield.

\section{INTRODUCTION}

Since 2014 sugar beet (Beta vulgaris, var. saccharifera) has become the main source for sugar production in Egypt due to the expansion of its area in a wide range of soils, i.e. saline, alkaline and calcareous. Nowadays, it occupies an important position among winter crops in the Egyptian crop rotation. Greater biomass of plant depends on the supply with environmental factors 
such as water, air temperature and carbon dioxide concentration in the canopy (Zbieć et al., 2003). The suitable sowing date of sugar beet in each region is influenced by the preceding crop, climate of the region, the convention contracted between farmers and sugar factory, in addition to the sown variety (Leilah et al. 2005). Osman et al. (2007) indicated that the earlier sowing date on September $15^{\text {th }}$ significantly attained the highest total soluble solids, sucrose and purity percentages, while juice impurities $\%$ was significantly reduced. Mosa (2009) revealed that early sowing sugar beet on 15 September increased root dimensions, sucrose and purity percentages, while impurities\% and sugar lost to molasses $\%$ were decreased. Also, yields of top, root and sugar were gradually decreased due to delaying sowing. Hemayati et al. (2012) showed that the highest root and white sugar yields were obtained by early sowing in September compared to delaying sowing. llkaee et al. (2016) reported that varying sowing date significantly affected root sugar $\%$.

Little attention has been directed for the role of carbon fixation in higher plants. Today, in order to achieve this goal, compounds such as methanol are sprayed to increase crop capability in $\mathrm{CO}_{2}$ fixation per unit area. Benson and Nonomura (1992) and Zbiec et al. (2003) found that methanol application had increased root yield by $23 \%$ compared to zero application (control). They added that the application of methanol at 20$30 \%(\mathrm{v} / \mathrm{v})$ increased root yield by $10 \%$. Abd El-Maged et al. (2004) found that sugar beet plants treated with methanol increased photosynthesis and yields of roots and sugar. Nadali et al. (2010) indicated that the application of $21 \%$ methanol solution increased fresh weights of root and leaf as well as sugar yield. However, foliar application of $14 \%$ methanol resulted in a maximum white sugar yield. Abido (2012) indicated that foliar application of $30 \%$ methanol solution led to significant increases in length and diameter of roots, foliage and root fresh weights, total chlorophyll, leaf area/plant, sucrose $\%$, purity $\%$ and yields of root, top and sugar. On the contrary, Khazaei et al. (2015) found that foliar application of methanol with 0 and $20 \%$ had insignificant effect on any measured traits of sugar beet.

The requirement of boron for plant growth was first discovered in the beginning of the $20^{\text {th }}$ century, and nowadays it is widely known that boron is an essential element for all vascular plants whose deficiency or toxicity causes impairments in several metabolic and physiological processes (Nable et al., 1997 and Blevins and Lukaszewski, 1998). Root dimensions, root fresh weight, sucrose \%, purity\% and root, top and sugar yields were significantly increased by increasing boron levels up to 2 kg/acre (Gobarah and Mekki, 2005). Dordas et al. (2007) reported that foliar application of $0.5 \mathrm{~kg} \mathrm{B/ha}$ increased $B$ concentration in leaves of sugar beet and hence led to the best quality and yields. Mohammad and Mohammad (2011) mentioned that spraying beets with $12 \%$ boric acid led to achieve a significant increase in yield and quality. Also, Abido (2012) cleared that increasing the application of boron significantly improved root yield and quality attributes of sugar beet. Armin and Asgharipour (2012) found that increasing boron levels up to $1.22 \mathrm{~kg}$ $\mathrm{B} / \mathrm{ha}$ led to increases in root yield and sucrose $\%$, while $\mathrm{K}, \mathrm{Na}$, $\alpha$-amino- $\mathrm{N}$, while molasses sugar were decreased compared to the control. El-Geddawy and Makhlouf (2015) found that increasing boron levels up to $210 \mathrm{ppm}$ caused significant increases in length, diameter and fresh weight of roots, sucrose $\%$, purity $\%$, yields of root, top and sugar/fed and boron content in root.

This work was conducted to find out the optimal sowing dates, methanol and boron levels to attain the maximum root and sugar yields with the best quality traits of sugar beet crop grown. 


\section{MATERIALS AND METHODS}

Two field experiments were conducted in Senouris, Fayoum Governorate, Egypt (latitude of $30.82^{\circ} \mathrm{N}$ and longitude of $29.40^{\circ}$ E) in $2014 / 2015$ and $2015 / 2016$ seasons to find out the optimal sowing date, methanol and boron fertilization levels to get the highest yield and quality of sugar beet. This work included two sowing dates $\left(15^{\text {th }}\right.$ September and $15^{\text {th }}$ October), three foliar concentrations of methanol $(0,10$ and $20 \%)$ and three foliar concentrations of boron $(0$, 0.5 and $1.0 \mathrm{~g}$ boric acid"17\% B"/l). Each solution of methanol contained $0.2 \%$ glycine to avoid the probability of methanol toxicity according to Nonomura and Benson (1992). Methanol solution was sprayed on sugar beet foliage three times. The $1^{\text {st }}$ dose was applied after 60 days from sowing, while the other two ones were applied at 15-day intervals. Boron levels were sprayed with the last methanol application. The volume of each solution was $300 \mathrm{l} / \mathrm{fed} " \mathrm{fed}^{-1}=0.42 \mathrm{ha}^{-1}$ ". At each sowing date, the nine combinations of methanol and boron levels were randomly distributed in a randomized complete block design with three replications. Thereafter, a combined analysis between the two sowing dates was done. Plot area was $21 \mathrm{~m}^{2}$ including 6 ridges of $50 \mathrm{~cm}$ in width; which were $7 \mathrm{~m}$ in length, where beet seeds were sown in hills of $20 \mathrm{~cm}$. Sugar beet Sara multi-germ variety was sown in both seasons. The preceding summer crop was sorghum in both seasons. Recommended doses of NPK were added. Nitrogen fertilizer was applied at $80 \mathrm{~kg} \mathrm{~N} / \mathrm{fed}$ as urea $(46.5 \%$ $N)$ in two equal doses, after thinning and month later. Phosphorus fertilizer was applied in form of calcium superphosphate $\left(\begin{array}{lll}15 \% & \mathrm{P}_{2} \mathrm{O}_{5}\end{array}\right)$ at $30 \mathrm{~kg} \mathrm{P}_{2} \mathrm{O}_{5} / \mathrm{fed}$ during seedbed preparation, whereas potassium fertilizer was added at $24 \mathrm{~kg} \mathrm{~K} \mathrm{~K}_{2} \mathrm{O} / \mathrm{fed}$ in form of potassium sulphate $\left(48 \% \mathrm{~K}_{2} \mathrm{O}\right)$ with the $2^{\text {nd }}$ nitrogen dose. Harvesting took place 210 days after sowing in both seasons. The rest of agricultural practices were followed as recommended by Sugar Crops Research Institute.

Soil samples were taken at random from the experimental sites at a depth of $0-30 \mathrm{~cm}$ from soil surface. The analyses of soil samples are presented in Table 1, which were done according to Piper (1950), Chapman and Pratt (1961), Jackson (1967), Markus et al. (1982) and Soltanpour (1991). Some metrological data of the experimental sites are presented in Table 2.

Table 1: Soil physical and chemical properties of the experimental sites

\begin{tabular}{|c|c|c|c|c|c|c|c|c|c|c|c|}
\hline \multirow{2}{*}{ Seasons } & \multicolumn{6}{|c|}{ Particle size distribution } & \multirow{2}{*}{\multicolumn{2}{|c|}{ Soil texture }} & \multirow{2}{*}{$\begin{array}{c}E C \\
\left(\mathrm{dsm}^{-1}\right)\end{array}$} & \multirow{2}{*}{$\begin{array}{c}\mathrm{pH} \\
(1: 2.5)\end{array}$} & \multirow{2}{*}{$\mathrm{SP} \%$} \\
\hline & \multicolumn{2}{|c|}{ Sand $\%$} & \multicolumn{2}{|c|}{ Silt \% } & \multicolumn{2}{|c|}{ Clay \% } & & & & & \\
\hline $2014 / 15$ & \multicolumn{2}{|c|}{24.1} & \multicolumn{2}{|c|}{36.6} & \multicolumn{2}{|c|}{39.3} & \multicolumn{2}{|c|}{ Clay loam } & 3.43 & 8.31 & 70.0 \\
\hline $2015 / 16$ & \multicolumn{2}{|c|}{25.5} & \multicolumn{2}{|c|}{37.6} & \multicolumn{2}{|c|}{36.9} & \multicolumn{2}{|c|}{ Clay loam } & 3.71 & 8.29 & 60.0 \\
\hline \multirow[t]{2}{*}{ Seasons } & \multicolumn{4}{|c|}{ Soluble cations $\left(\mathrm{mq} \mathrm{l}^{-1}\right)$} & \multicolumn{3}{|c|}{ Soluble ions $\left(\mathrm{mq} \mathrm{l}^{-1}\right)$} & \multirow{2}{*}{$\begin{array}{c}\text { B } \\
\mathrm{ppm}\end{array}$} & \multicolumn{3}{|c|}{$\begin{array}{l}\text { Available nutrients } \\
\text { (mg/1kg soil }))\end{array}$} \\
\hline & $\mathrm{Ca}^{++}$ & $\mathrm{Mg}^{++}$ & $\mathrm{Na}^{+}$ & $\mathrm{K}^{+}$ & $\mathrm{HCO}_{3}^{-}$ & $\mathrm{Cl}^{-}$ & $\mathrm{SO}_{4}^{--}$ & & $\mathrm{N}$ & $P$ & $\mathrm{~K}$ \\
\hline $2014 / 15$ & 9.8 & 5.55 & 18.3 & 0.65 & 2.5 & 26.1 & 5.7 & 0.022 & 52.3 & 5.17 & 142 \\
\hline $2015 / 16$ & 11.3 & 5.64 & 19.7 & 0.42 & 2.8 & 29.2 & 5.1 & 0.038 & 54.6 & 5.42 & 148 \\
\hline
\end{tabular}


Table 2: Some metrological data of the experimental sites.

\begin{tabular}{|c|c|c|c|c|c|c|}
\hline \multirow{3}{*}{ Months } & \multicolumn{3}{|c|}{$2014 / 2015$} & \multicolumn{3}{|c|}{$2015 / 2016$} \\
\hline & \multicolumn{2}{|c|}{$\begin{array}{c}\text { Air } \\
\text { temperature }{ }^{\circ} \mathrm{C}\end{array}$} & \multirow{2}{*}{$\begin{array}{c}\text { Relative } \\
\text { humidity \% }\end{array}$} & \multicolumn{2}{|c|}{$\begin{array}{c}\text { Air } \\
\text { temperature }{ }^{\circ} \mathrm{C}\end{array}$} & \multirow{2}{*}{$\begin{array}{c}\text { Relative } \\
\text { humidity \% }\end{array}$} \\
\hline & Max. & Min. & & Max. & Min. & \\
\hline September & 38.4 & 24.4 & 45.9 & 37.7 & 23.4 & 47.0 \\
\hline October & 34.8 & 21.5 & 47.7 & 32.7 & 20.7 & 57.0 \\
\hline November & 29.3 & 17.2 & 45.4 & 26.9 & 15.7 & 46.0 \\
\hline December & 26.1 & 12.6 & 45.8 & 21.6 & 9.9 & 64.7 \\
\hline January & 22.5 & 10.3 & 45.9 & 19.6 & 8.3 & 60.3 \\
\hline February & 23.6 & 10.5 & 49.0 & 24.4 & 10.4 & 54.0 \\
\hline March & 28.8 & 14.9 & 47.9 & 27.3 & 13.1 & 43.3 \\
\hline April & 32.8 & 15.7 & 45.0 & 33.5 & 16.5 & 38.3 \\
\hline May & 37.9 & 21.9 & 46.6 & 35.4 & 18.1 & 42.1 \\
\hline
\end{tabular}

Source: Agro-meteorological Station, Agric. Res. Center, Giza, Egypt.

\section{The recorded data:}

Ten plants were taken at random from the guarded ridges of each plot during the growth period, after 20 days from the last foliar application to determinate the following traits:

1. Leaf area index (LAl) was determined using the disk method, using 10 disks of $1.0 \mathrm{~cm}$ diameter according to the method described by Watson (1958) and then the following equation was used:

LAI $=$ leaf area per plant $\left(\mathrm{cm}^{2}\right) /$ plant ground area $\left(\mathrm{cm}^{2}\right)$.

2. Net assimilation rate (NAR) was measured according to the method shown by Radford's (1967) using the following equation:

$\mathrm{NAR}=\frac{\left(W_{2}-W_{1}\right)\left(\log _{e} A_{2}-\log _{e} A_{1}\right)}{\left(T_{2}-T_{1}\right)\left(A_{2}-A_{1}\right)} \mathrm{g} / \mathrm{m}^{2} /$ day

Where: $W_{1}, A_{1}$ and $W_{2}, A_{2}$, respectively refer to dry weight and leaf area of plant at sampling time $T_{1}$ and $T_{2}$. (30-day interval).

3. Photosynthetic pigments were determined in the fresh leaves according as shown by Wettestien (1957) using the following equations:
Chl. "a" mg/g.f.w. = 9.684 (A 662) -0.99 (A 644).

Chl. "b" mg/g.f.w. $=21.426$ (A 644) -4.65 (A 662).

Carot. $\quad \mathrm{mg} / \mathrm{g}$.f.w. $=4.695$ (A 440) -0.268 ( chl. "a" + chl. "b").

Where; chl. "a", "b" and carot. = concentrations of chlorophylls "a", "b" and carotenoids, respectively, and $A=$ optical density at the wave length indicated.

At harvest, ten plants were taken at random from the guarded ridges of each plot to determine the following characteristics:

1. Root length $(\mathrm{cm})$.

2. Root diameter $(\mathrm{cm})$.

3. Root fresh weight (g/plant).

4. Sucrose \% was determined as reported by Le Docte (1927).

5. Purity \% was calculated according to the equation of Deviller (1988) as follows:

Purity $\%=99.36-[14.27(\mathrm{Na}+\mathrm{K}+$ a-amino N) / sucrose\%].

6. Sugar lost to molasses (SLM) was calculated according to the equation of Deviller (1988) as follows:

$S L M=0.14(\mathrm{Na}+\mathrm{K})+0.25(a-a \operatorname{mino} \mathrm{N})+0.5$ 
7. Extractable sugar\% (ES\%) was calculated according to Dexter et al. (1967) as follows: $E S \%=$ sucrose $\%-S L M-0.6$

8. Potassium, sodium and a-amino $N$ concentrations of juice were determined in Fayoum Sugar Company Laboratories.

9. The concentration of phenolics in leaves extracts was determined using spectrophotometric method (Singleton et al., 1999).

10. Crude fiber was determined as described in A.O.A.C. (2005).

Plants of each plot were uprooted, topped, cleaned and weighed to determine the following parameters:

1. Root yield (ton/fed).

2. Top yield (ton/fed).

3. Sugar yield $($ ton/fed $)=$ extractable sugar\% $\mathrm{x}$ root yield (ton/fed).

\section{Statistical analysis:}

The collected data were statistically analyzed as illustrated by Snedecor and Cochran (1981). Least Significant Difference (LSD) was used to compare the differences between means at $5 \%$ level of probability as mentioned by Waller and Duncan (1969).

\section{RESULTS AND DISCUSSION}

\section{A. Agronomical and physiological criteria:}

1. Root length, root diameter and root fresh weight/plant:

Results in Table 3 clear that sugar beet sown earlier on September $15^{\text {th }}$ significantly surpassed that planted later on October $15^{\text {th }}$ in root length, diameter and fresh weight/plant, in both seasons. The superiority of planting sugar beet on $15^{\text {th }}$ September with respect to root fresh weight may be due to favorable weather conditions during the growing season, which ensured rapid growth and formation a good canopy of beet plants, reflected on an efficient photosynthesis and hence resulted in maximum growth and storage of dry matter in roots. These results are in harmony with those obtained by Mosa (2009).
Regarding methanol effect, data in Table 3 pointed a significant and positive response of these traits to the sprayed methanol levels. These results are in line with those confirmed by Nadali et al. (2010) and Abido (2012). Increasing the concentration of methanol solution to $20 \%$ caused an increase in root length amounted to 2.52 and $2.84 \mathrm{~cm}$, corresponding to 1.94 and $0.62 \mathrm{~cm}$ in root diameter as well as 259 and $120 \mathrm{~g}$ in root fresh weight/plant, in the $1^{\text {st }}$ and $2^{\text {nd }}$ season, respectively, compared to the check treatment. These increments may be due to the effect of methanol in increasing photosynthesis with delaying leaf senescence and affecting rate of ethylene production, which finally participated in increasing root size.

Data in Table 3 show that the gradual increase in the sprayed concentrations of boron on sugar beet foliage up to $1.0 \mathrm{~g}$ boric acid/l significantly increased root dimensions as well as root fresh weight/plant. These observations were true in both seasons. The positive effect of boron may be due to its effective role in cell elongation of root. These results are in harmony with those obtained by Gobarah and Mekki (2005) and El-Geddawy and Makhlouf (2015).

Regarding the $1^{\text {st }}$ order interaction effects between the studied factors, results in Table 3 pointed out that root length was significantly influenced by the interaction between sowing dates and methanol concentrations in both seasons. The same interaction had a significant effect on root fresh weight/plant, in the $1^{\text {st }}$ season only. The interaction between sowing dates and boron concentrations significantly affected both root length and fresh weight in the $2^{\text {nd }}$ season. It was generally noticed that sowing sugar beet earlier on 15 September combined with raising the concentration of the sprayed solution of each of methanol and boron resulted in higher values of root length and fresh weight. 
Table 3: Root length, diameter and fresh weight/plant of sugar beet as affected by sowing date, methanol and boron foliar application in 2014/2015 and 2015/2016 seasons

\begin{tabular}{|c|c|c|c|c|c|c|c|c|c|}
\hline \multirow{2}{*}{\multicolumn{2}{|c|}{ Treatments }} & \multicolumn{8}{|c|}{ Root length (cm) } \\
\hline & & \multicolumn{4}{|c|}{$2014 / 2015$} & \multicolumn{4}{|c|}{$2015 / 2016$} \\
\hline \multirow{2}{*}{$\begin{array}{l}\text { Sowing } \\
\text { dates }\end{array}$} & \multirow{2}{*}{$\begin{array}{c}\text { Methanol } \\
\text { levels }\end{array}$} & \multicolumn{8}{|c|}{ Boron levels ( $\mathrm{g}$ boric $\mathrm{acid} / \mathrm{l})$} \\
\hline & & 0 & 0.5 & 1.0 & Mean & 0 & 0.5 & 1.0 & Mean \\
\hline & 0 & 23.67 & 24.67 & 26.67 & 25.00 & 22.89 & 24.00 & 26.22 & 24.37 \\
\hline Sentember & $10 \%$ & 25.00 & 26.78 & 28.11 & 26.63 & 23.11 & 24.78 & 26.67 & 24.85 \\
\hline sepiemier & $20 \%$ & 25.11 & 26.33 & 28.56 & 26.67 & 24.83 & 26.78 & 29.22 & 26.95 \\
\hline \multicolumn{2}{|c|}{ Mean } & 24.59 & 25.93 & 27.78 & 26.10 & 23.61 & 25.19 & 27.37 & 25.39 \\
\hline & 0 & 20.11 & 22.11 & 24.78 & 22.33 & 20.89 & 22.89 & 23.33 & 22.37 \\
\hline & $10 \%$ & 22.67 & 23.11 & 25.89 & 23.89 & 21.67 & 25.33 & 25.56 & 24.19 \\
\hline uctoder & $20 \%$ & 23.67 & 25.33 & 28.11 & 25.70 & 23.78 & 25.45 & 27.22 & 25.48 \\
\hline \multicolumn{2}{|c|}{ Mean } & 22.15 & 23.52 & 26.26 & 23.98 & 22.11 & 24.56 & 25.37 & 24.01 \\
\hline Methanol & 0 & 21.89 & 23.39 & 25.72 & 23.67 & 21.89 & 23.45 & 24.78 & 23.37 \\
\hline $\mathrm{x}$ & $10 \%$ & 23.83 & 24.94 & 27.00 & 25.26 & 22.39 & 25.06 & 26.11 & 24.52 \\
\hline Boron & $20 \%$ & 24.39 & 25.83 & 28.33 & 26.19 & 24.31 & 26.11 & 28.22 & 26.21 \\
\hline \multicolumn{2}{|c|}{ Mean } & 23.37 & 24.72 & 27.02 & & 22.86 & 24.87 & 26.37 & \\
\hline \multicolumn{10}{|c|}{ LSD at 0.05 level for: } \\
\hline \multirow{4}{*}{\multicolumn{2}{|c|}{$\begin{array}{l}\text { Sowing dates }(A) \\
\text { Methanol levels }(B) \\
\text { Boron levels }(C) \\
A \times B\end{array}$}} & \multicolumn{2}{|c|}{0.36} & $A \times C$ & NS & A & 0.43 & $A \times C$ & 0.75 \\
\hline & & \multicolumn{2}{|c|}{0.44} & $\mathrm{~B} \times \mathrm{C}$ & NS & B & 0.53 & $\mathrm{~B} \times \mathrm{C}$ & NS \\
\hline & & \multicolumn{2}{|c|}{0.44} & $\mathrm{AxB} \times \mathrm{C}$ & NS & C & 0.53 & $\mathrm{AxB} \times \mathrm{C}$ & NS \\
\hline & & \multicolumn{2}{|c|}{0.63} & & & $A \times B$ & 0.75 & & \\
\hline & & & & & Toot dia & ter $(\mathrm{cm}$ & & & \\
\hline & 0 & 11.00 & 11.44 & 12.45 & 11.63 & 10.78 & 11.67 & 11.92 & 11.46 \\
\hline Sentemb & $10 \%$ & 11.44 & 12.56 & 13.33 & 12.44 & 11.67 & 12.00 & 12.29 & 11.99 \\
\hline September & $20 \%$ & 12.44 & 13.67 & 13.67 & 13.26 & 11.56 & 12.11 & 12.45 & 12.04 \\
\hline & & 11.63 & 12.56 & 13.15 & 12.44 & 11.33 & 11.93 & 12.22 & 11.83 \\
\hline & 0 & 9.07 & 9.68 & 10.57 & 9.77 & 9.89 & 10.00 & 10.44 & 10.11 \\
\hline 15 & $10 \%$ & 10.23 & 11.01 & 11.68 & 10.97 & 9.89 & 10.67 & 11.14 & 10.57 \\
\hline October & $20 \%$ & 11.68 & 12.01 & 12.57 & 12.09 & 10.11 & 10.72 & 11.42 & 10.75 \\
\hline & & 10.33 & 10.90 & 11.60 & 10.94 & 9.96 & 10.46 & 11.00 & 10.48 \\
\hline Methanol & 0 & 10.03 & 10.56 & 11.51 & 10.70 & 10.33 & 10.84 & 11.18 & 10.78 \\
\hline $\mathrm{x}$ & $10 \%$ & 10.84 & 11.78 & 12.51 & 11.71 & 10.78 & 11.33 & 11.72 & 11.28 \\
\hline Boron & $20 \%$ & 12.06 & 12.84 & 13.12 & 12.67 & 10.83 & 11.42 & 11.94 & 11.40 \\
\hline & & & 11.73 & 12.38 & & 10.65 & 11.20 & 11.61 & \\
\hline LSD at 0.05 & for: & & & & & & & & \\
\hline Sowing date & & & & $A \times C$ & NS & $A$ & 0.30 & $A \times C$ & NS \\
\hline Methanol le & & & & $B \times C$ & NS & B & 0.37 & $B \times C$ & NS \\
\hline Boron levels & & & & $\mathrm{A} \times \mathrm{B} \times \mathrm{C}$ & NS & C & 0.37 & $\mathrm{AxB} \times \mathrm{C}$ & NS \\
\hline & & & & & & $A \times B$ & NS & & \\
\hline & & & & Roc & fresh w & $\mathrm{ght}(\mathrm{kg} /$ & ant) & & \\
\hline $15^{\text {th }}$ & 0 & 0.951 & 1.007 & 1.057 & 1.005 & 0.750 & 0.927 & 0.997 & 0.891 \\
\hline Septemb & $10 \%$ & 0.985 & 1.058 & 1.143 & 1.062 & 0.695 & 1.062 & 1.072 & 0.943 \\
\hline September & $20 \%$ & 1.167 & 1.223 & 1.280 & 1.223 & 0.876 & 1.037 & 1.162 & 1.025 \\
\hline & & 1.034 & 1.096 & 1.160 & 1.097 & 0.774 & 1.009 & 1.077 & 0.953 \\
\hline $15^{\text {th }}$ & 0 & 0.663 & 0.765 & 0.922 & 0.784 & 0.660 & 0.851 & 0.937 & 0.816 \\
\hline $15^{\text {tin }}$ & $10 \%$ & 0.842 & 0.913 & 0.980 & 0.912 & 0.798 & 0.950 & 0.940 & 0.896 \\
\hline October & $20 \%$ & 0.997 & 1.083 & 1.167 & 1.082 & 0.877 & 0.941 & 0.951 & 0.923 \\
\hline & & 0.834 & 0.921 & 1.023 & 0.926 & 0.778 & 0.914 & 0.943 & 0.878 \\
\hline Methanol & 0 & 0.807 & 0.886 & 0.990 & 0.894 & 0.705 & 0.889 & 0.967 & 0.854 \\
\hline x & $10 \%$ & 0.914 & 0.986 & 1.062 & 0.987 & 0.747 & 1.006 & 1.006 & 0.920 \\
\hline Boron & $20 \%$ & 1.082 & 1.153 & 1.223 & 1.153 & 0.877 & 0.989 & 1.057 & 0.974 \\
\hline & & 0.934 & 1.008 & 1.092 & & 0.776 & 0.961 & 1.010 & \\
\hline LSD at 0.05 & for: & & & & & & & & \\
\hline Sowing date & & & & $A \times C$ & NS & A & 0.037 & $A \times C$ & 0.064 \\
\hline Methanol lev & (B) & & & $B \times C$ & NS & B & 0.045 & $B \times C$ & NS \\
\hline Boron levels & & & & $\mathrm{A} \times \mathrm{B} \times \mathrm{C}$ & NS & C & 0.045 & $\mathrm{~A} \times \mathrm{B} \times \mathrm{C}$ & NS \\
\hline$A \times B$ & & & & & & $A \times B$ & NS & & \\
\hline
\end{tabular}




\section{Leaf area index (LAI) and net assimilation rate (NAR):}

Data in Table 4 clear that planting sugar beet earlier on 15 September significantly resulted in higher values of LAl and NAR than that sown on 15 October in the $1^{\text {st }}$ and $2^{\text {nd }}$ seasons. The distinct effect of earlier sowing dates on these traits is mainly due to the favourable climatic conditions especially the temperature degree and light intensity which accelerated vegetative growth, formation of good canopy capable to increase photosynthesis process. These results were partially agreed with those reported by Mosa (2009).

Increasing methanol concentrations from zero up to $20 \%$ led to significant, gradual and positive increases in LAI and NAR. These results could be referred to the role of methanol in delaying senescence of leaves and influencing ethylene production in plant, which may increase photosynthesis activity (Zbiec et al., 1999).

There was a significant and continuous response in LAI and NAR with increasing the applied dose of boron fertilizer. Foliar spraying of boron at $1.0 \mathrm{~g}$ boric acid/l recorded the highest values of these traits, in the two growing seasons. The advantage of boron application may be due to the function of boron in increasing plant metabolism, development and growth (Abido, 2012).

The interaction between sowing date and methanol application significantly affected LAI and NAR, in both seasons. Sowing sugar beet on 15 September achieved the highest values in LAI and NAR compared to sowing on 15 October, when plants were sprayed with $20 \%$ methanol solution in both seasons. The interaction between the levels of methanol and boron significantly affected LAI, in the $1^{\text {st }}$ season and NAR, in the $2^{\text {nd }}$ one.

\section{Photosynthetic pigments:}

Leaf pigments substances refer to the contents of chlorophyll "a", "b" and carotenoids. Data in Table 5 affirmed that the sowing dates attained significant effects on chlorophyll "a" in both seasons, as well as chlorophyll "b" and carotenoids in the $1^{\text {st }}$ season only. Results pointed out that earlier sowing of sugar beet on 15 September significantly increased the photosynthetic pigments. The increments in photosynthetic pigments accompanied the earlier planting might be ascribed to more suitable weather conditions in respect to temperature and light intensity, which assured better establishment and growth criteria.

Significant effects on photosynthetic pigments were noticed, in both seasons (Table 5). Results showed that increasing methanol levels up to $20 \%$ led to significant increases in chlorophyll "a" and "b" as well as carotenoids. Benson and Nonomura (1992) explained that the stimulatory effect of methanol on the growth of plant biomass is based on the increase in chlorophyll pigments substance activity, which results in efficient use of photosynthesis for energy storage into biomass.

Table 5 showed that increasing boron levels up to $1.0 \mathrm{~g}$ boric acid/l led to significant increases in chlorophyll "a" and "b" as well as carotenoids in both seasons. The advantage of boron application may be due to its important function in increasing plant metabolism, development and growth. These results are in line with those confirmed by Abido (2012).

The interaction between sowing dates and methanol applications caused significant effects on chlorophyll "a" and carotinoids in both seasons. Raising methanol levels up to $20 \%$, when sugar beet was planted on the $15^{\text {th }}$ of September led to the highest chlorophyll "a" and "b" as well as carotinoids compared to sowing on 15 October. Meantime, the combination between methanol and boron applications caused significant effects in the values of chlorophyll "b" in both seasons. 
Table 4: Leaf area index and net assimilation rate as affected by sowing date, methanol and boron foliar application and their interactions in 2014/2015 and 2015/2016 seasons

\begin{tabular}{|c|c|c|c|c|c|c|c|c|c|}
\hline \multirow{2}{*}{\multicolumn{2}{|c|}{ Treatments }} & \multicolumn{8}{|c|}{ Leaf area index (LAl) } \\
\hline & & \multicolumn{4}{|c|}{$2014 / 2015$} & \multicolumn{4}{|c|}{$2015 / 2016$} \\
\hline \multirow{2}{*}{$\begin{array}{l}\text { Sowing } \\
\text { dates }\end{array}$} & \multirow{2}{*}{$\begin{array}{c}\text { Methanol } \\
\text { levels }\end{array}$} & \multicolumn{8}{|c|}{ Boron levels ( $\mathrm{g}$ boric acid/l) } \\
\hline & & 0 & 0.5 & 1.0 & Mean & 0 & 0.5 & 1.0 & Mean \\
\hline \multirow{3}{*}{$\begin{array}{c}15^{\text {th }} \\
\text { September }\end{array}$} & 0 & 3.30 & 3.39 & 3.67 & 3.45 & 2.84 & 3.47 & 3.79 & 3.37 \\
\hline & $10 \%$ & 3.44 & 3.55 & 3.43 & 3.47 & 3.60 & 3.67 & 3.85 & 3.71 \\
\hline & $20 \%$ & 3.64 & 3.60 & 3.76 & 3.67 & 3.69 & 3.86 & 3.93 & 3.83 \\
\hline \multicolumn{2}{|c|}{ Mean } & 3.46 & 3.51 & 3.62 & 3.53 & 3.38 & 3.67 & 3.86 & 3.63 \\
\hline \multirow{3}{*}{$\begin{array}{c}15^{\text {th }} \\
\text { October }\end{array}$} & 0 & 2.84 & 3.00 & 3.08 & 2.97 & 2.25 & 2.49 & 2.94 & 2.56 \\
\hline & $10 \%$ & 3.22 & 3.33 & 3.42 & 3.32 & 3.01 & 3.21 & 3.47 & 3.23 \\
\hline & $20 \%$ & 3.49 & 3.44 & 3.60 & 3.51 & 3.23 & 3.48 & 3.63 & 3.45 \\
\hline \multicolumn{2}{|c|}{ Mean } & 3.18 & 3.26 & 3.37 & 3.27 & 2.83 & 3.06 & 3.35 & 3.08 \\
\hline Methanol & 0 & 3.07 & 3.20 & 3.37 & 3.21 & 2.55 & 2.98 & 3.36 & 2.97 \\
\hline $\mathrm{x}$ & $10 \%$ & 3.33 & 3.44 & 3.42 & 3.40 & 3.31 & 3.44 & 3.66 & 3.47 \\
\hline Boron & $20 \%$ & 3.57 & 3.52 & 3.68 & 3.59 & 3.46 & 3.67 & 3.78 & 3.64 \\
\hline \multicolumn{2}{|c|}{ Mean } & 3.32 & 3.38 & 3.49 & & 3.10 & 3.36 & 3.60 & \\
\hline
\end{tabular}

\begin{tabular}{|c|c|c|c|c|c|c|c|c|c|}
\hline \multicolumn{10}{|c|}{ LSD at 0.05 level for: } \\
\hline \multicolumn{2}{|c|}{ Sowing dates $(A)$} & \multicolumn{2}{|c|}{0.06} & $A \times C$ & NS & A & 0.14 & $A \times C$ & NS \\
\hline \multicolumn{2}{|c|}{ Methanol levels (B) } & \multicolumn{2}{|c|}{0.07} & $\mathrm{~B} \times \mathrm{C}$ & 0.12 & B & 0.17 & $B \times C$ & NS \\
\hline \multicolumn{2}{|c|}{ Boron levels $(\mathrm{C})$} & \multicolumn{2}{|c|}{0.07} & $A \times B \times C$ & NS & C & 0.17 & $\mathrm{AxB} \times \mathrm{C}$ & NS \\
\hline \multicolumn{2}{|l|}{$A \times B$} & \multicolumn{2}{|c|}{0.10} & & & $A \times B$ & 0.24 & & \\
\hline & & \multicolumn{8}{|c|}{ Net assimilation rate $\left(\mathrm{g} / \mathrm{m}^{2} /\right.$ day $)$} \\
\hline \multirow{3}{*}{$\begin{array}{c}15^{\text {th }} \\
\text { September }\end{array}$} & 0 & 3.10 & 3.11 & 3.24 & 3.15 & 3.32 & 3.51 & 3.63 & 3.49 \\
\hline & $10 \%$ & 3.36 & 3.39 & 3.53 & 3.43 & 3.72 & 3.78 & 3.89 & 3.80 \\
\hline & $20 \%$ & 3.52 & 3.75 & 3.89 & 3.72 & 3.85 & 3.88 & 4.14 & 3.96 \\
\hline \multicolumn{2}{|c|}{ Mean } & 3.33 & 3.42 & 3.55 & 3.43 & 3.63 & 3.72 & 3.89 & 3.75 \\
\hline \multirow{3}{*}{$\begin{array}{c}15^{\text {th }} \\
\text { October }\end{array}$} & 0 & 2.74 & 2.94 & 3.10 & 2.93 & 2.65 & 2.79 & 3.08 & 2.84 \\
\hline & $10 \%$ & 2.99 & 3.04 & 3.58 & 3.20 & 3.23 & 3.34 & 3.45 & 3.34 \\
\hline & $20 \%$ & 3.05 & 3.18 & 3.46 & 3.23 & 3.40 & 3.42 & 3.54 & 3.45 \\
\hline \multicolumn{2}{|c|}{ Mean } & 2.93 & 3.06 & 3.38 & 3.12 & 3.09 & 3.18 & 3.36 & 3.21 \\
\hline Methanol & 0 & 2.92 & 3.03 & 3.17 & 3.04 & 2.98 & 3.15 & 3.36 & 3.16 \\
\hline $\mathrm{x}$ & $10 \%$ & 3.18 & 3.22 & 3.55 & 3.32 & 3.48 & 3.56 & 3.67 & 3.57 \\
\hline Boron & $20 \%$ & 3.28 & 3.47 & 3.67 & 3.48 & 3.63 & 3.65 & 3.84 & 3.71 \\
\hline \multicolumn{2}{|c|}{ Mean } & 3.13 & 3.24 & 3.46 & & 3.36 & 3.45 & 3.62 & \\
\hline \multicolumn{10}{|c|}{ LSD at 0.05 level for: } \\
\hline \multicolumn{2}{|c|}{ Sowing dates $(A)$} & \multicolumn{2}{|c|}{0.09} & $A \times C$ & NS & A & 0.04 & $A \times C$ & NS \\
\hline \multicolumn{2}{|c|}{ Methanol levels (B) } & \multicolumn{2}{|c|}{0.11} & $B \times C$ & NS & B & 0.05 & $B \times C$ & 0.09 \\
\hline \multicolumn{2}{|c|}{ Boron levels (C) } & \multicolumn{2}{|c|}{0.11} & $A \times B \times C$ & NS & C & 0.05 & $\mathrm{AxB} \times \mathrm{C}$ & NS \\
\hline \multicolumn{2}{|l|}{$A \times B$} & \multicolumn{2}{|c|}{0.16} & & & $A \times B$ & 0.07 & & \\
\hline
\end{tabular}


Table 5: Photosynthetic pigments as affected by sowing date, methanol and boron foliar application and their interactions in 2014/2015 and 2015/2016 seasons

\begin{tabular}{|c|c|c|c|c|c|c|c|c|c|}
\hline \multirow{2}{*}{\multicolumn{2}{|c|}{ Treatments }} & \multicolumn{8}{|c|}{ Chlorophyll "a" (mg/g.f.w) } \\
\hline & & \multicolumn{4}{|c|}{$2014 / 2015$} & \multicolumn{4}{|c|}{$2015 / 2016$} \\
\hline \multirow{2}{*}{$\begin{array}{l}\text { Sowing } \\
\text { dates }\end{array}$} & \multirow{2}{*}{$\begin{array}{c}\text { Methanol } \\
\text { levels }\end{array}$} & \multicolumn{8}{|c|}{ Boron levels (g boric acid/l) } \\
\hline & & 0 & 0.5 & 1.0 & Mean & 0 & 0.5 & 1.0 & Mean \\
\hline $15^{\text {th }}$ & 0 & 4.26 & 4.30 & 4.45 & 4.34 & 3.72 & 3.74 & 4.47 & 3.98 \\
\hline Sentember & $10 \%$ & 4.73 & 4.88 & 5.34 & 4.98 & 4.80 & 4.87 & 5.00 & 4.89 \\
\hline September & $20 \%$ & 5.59 & 5.66 & 5.78 & 5.68 & 5.32 & 5.50 & 5.87 & 5.56 \\
\hline \multicolumn{2}{|c|}{ Mean } & 4.86 & 4.95 & 5.19 & 5.00 & 4.61 & 4.70 & 5.11 & 4.81 \\
\hline & 0 & 2.92 & 3.12 & 3.35 & 3.13 & 3.06 & 3.37 & 3.46 & 3.30 \\
\hline 15 & $10 \%$ & 3.97 & 4.10 & 4.18 & 4.08 & 4.39 & 4.49 & 4.59 & 4.49 \\
\hline & $20 \%$ & 4.22 & 4.23 & 4.40 & 4.28 & 4.65 & 4.66 & 4.83 & 4.71 \\
\hline \multicolumn{2}{|c|}{ Mean } & 3.70 & 3.82 & 3.98 & 3.83 & 4.03 & 4.17 & 4.29 & 4.17 \\
\hline Methanol & 0 & 3.59 & 3.71 & 3.90 & 3.74 & 3.39 & 3.55 & 3.97 & 3.64 \\
\hline $\mathrm{x}$ & $10 \%$ & 4.35 & 4.49 & 4.76 & 4.53 & 4.59 & 4.68 & 4.80 & 4.69 \\
\hline Boron & $20 \%$ & 4.91 & 4.95 & 5.09 & 4.98 & 4.99 & 5.08 & 5.35 & 5.14 \\
\hline \multicolumn{2}{|c|}{ Mean } & 4.28 & 4.38 & 4.58 & & 4.32 & 4.44 & 4.70 & \\
\hline \multicolumn{10}{|c|}{ LSD at 0.05 level for: } \\
\hline \multicolumn{2}{|c|}{ Sowing dates $(A)$} & \multicolumn{2}{|c|}{0.16} & $A \times C$ & NS & A & 0.10 & $A \times C$ & 0.17 \\
\hline \multicolumn{2}{|c|}{ Methanol levels (B) } & \multicolumn{2}{|c|}{0.19} & $B \times C$ & NS & $\mathrm{B}$ & 0.12 & $\mathrm{~B} \times \mathrm{C}$ & NS \\
\hline \multicolumn{2}{|c|}{ Boron levels $(\mathrm{C})$} & & & $\mathrm{A} \times \mathrm{B} \times \mathrm{C}$ & NS & $\mathrm{C}$ & 0.12 & $\mathrm{~A} \times \mathrm{B} \times \mathrm{C}$ & NS \\
\hline$A \times B$ & & & & & & $A \times B$ & 0.17 & & \\
\hline & & & & & rophyll & $"(\mathrm{mg} / \mathrm{g}$ & & & \\
\hline & 0 & 1.85 & 2.27 & 2.51 & 2.21 & 1.98 & 2.68 & 2.87 & 2.51 \\
\hline $15^{\text {tit }}$ & $10 \%$ & 2.81 & 3.05 & 3.25 & 3.04 & 2.77 & 3.03 & 3.12 & 2.97 \\
\hline seplemioer & $20 \%$ & 3.16 & 3.26 & 3.64 & 3.35 & 2.93 & 3.04 & 3.21 & 3.06 \\
\hline & & 2.61 & 2.86 & 3.13 & 2.87 & 2.56 & 2.92 & 3.07 & 2.85 \\
\hline $15^{\text {th }}$ & 0 & 1.52 & 1.81 & 2.04 & 1.79 & 2.00 & 2.24 & 2.72 & 2.32 \\
\hline October & $10 \%$ & 2.26 & 2.55 & 2.73 & 2.51 & 2.33 & 2.54 & 2.67 & 2.51 \\
\hline & $20 \%$ & 2.59 & 2.51 & 2.95 & 2.68 & 2.46 & 2.78 & 2.96 & 2.74 \\
\hline & & 2.12 & 2.29 & 2.57 & 2.33 & 2.26 & 2.52 & 2.79 & 2.52 \\
\hline Methanol & 0 & 1.69 & 2.04 & 2.27 & 2.00 & 1.99 & 2.46 & 2.80 & 2.41 \\
\hline $\mathrm{x}$ & $10 \%$ & 2.53 & 2.80 & 2.99 & 2.77 & 2.55 & 2.79 & 2.90 & 2.74 \\
\hline Boron & $20 \%$ & 2.87 & 2.88 & 3.29 & 3.02 & 2.70 & 2.91 & 3.09 & 2.90 \\
\hline & & 2.36 & 2.57 & 2.85 & & 2.41 & 2.72 & 2.93 & \\
\hline LSD at 0.05 & el for: & & & & & & & & \\
\hline Sowing date & & & & $A \times C$ & NS & A & NS & $A \times C$ & NS \\
\hline Methanol lev & (B) & & & $B \times C$ & 0.10 & $B$ & 0.11 & $B \times C$ & 0.20 \\
\hline Boron levels & & & & $\mathrm{A} \times \mathrm{B} \times \mathrm{C}$ & NS & C & 0.11 & $\mathrm{AxB} \times \mathrm{C}$ & NS \\
\hline$A \times B$ & & & & & & $A \times B$ & NS & & \\
\hline & & & & & rotenoi & $(\mathrm{mg} / \mathrm{g} . \mathrm{f}$ & & & \\
\hline & 0 & 0.88 & 0.91 & 1.05 & 0.95 & 1.19 & 1.33 & 1.53 & 1.35 \\
\hline $\begin{array}{c}15 \\
\text { Sentemb }\end{array}$ & $10 \%$ & 1.32 & 1.36 & 1.82 & 1.50 & 1.52 & 1.58 & 1.72 & 1.61 \\
\hline septemioer & $20 \%$ & 1.95 & 2.13 & 2.25 & 2.11 & 1.97 & 2.16 & 2.33 & 2.15 \\
\hline & & 1.38 & 1.47 & 1.71 & 1.52 & 1.56 & 1.69 & 1.86 & 1.70 \\
\hline $15^{\text {th }}$ & 0 & 0.93 & 1.10 & 1.47 & 1.17 & 0.87 & 1.07 & 1.18 & 1.04 \\
\hline $15^{\text {tit }}$ & $10 \%$ & 0.96 & 1.08 & 1.57 & 1.20 & 1.16 & 1.18 & 1.30 & 1.21 \\
\hline October & $20 \%$ & 1.47 & 1.57 & 1.62 & 1.55 & 1.48 & 1.56 & 1.71 & 1.58 \\
\hline & & 1.12 & 1.25 & 1.55 & 1.31 & 1.17 & 1.27 & 1.39 & 1.28 \\
\hline Methanol & 0 & 0.91 & 1.01 & 1.26 & 1.06 & 1.03 & 1.20 & 1.35 & 1.19 \\
\hline $\mathrm{x}$ & $10 \%$ & 1.14 & 1.22 & 1.69 & 1.35 & 1.34 & 1.38 & 1.51 & 1.41 \\
\hline Boron & $20 \%$ & 1.71 & 1.85 & 1.93 & 1.83 & 1.72 & 1.86 & 2.02 & 1.87 \\
\hline & & 1.25 & 1.36 & 1.63 & & 1.36 & 1.48 & 1.63 & \\
\hline LSD at 0.05 & el for: & & & & & & & & \\
\hline Sowing date & & & & $A \times C$ & NS & A & NS & $A \times C$ & NS \\
\hline Methanol lev & (B) & & & $B \times C$ & NS & $B$ & 0.08 & $\mathrm{~B} \times \mathrm{C}$ & NS \\
\hline Boron levels & & & & $\mathrm{A} \times \mathrm{B} \times \mathrm{C}$ & NS & $\mathrm{C}$ & 0.08 & $\mathrm{~A} \times \mathrm{B} \times \mathrm{C}$ & NS \\
\hline$A \times B$ & & & & & & $A \times B$ & 0.11 & & \\
\hline
\end{tabular}




\section{Fiber content in roots and poly} phenol in leaves:

Results in Table 6 pointed out that earlier sowing of sugar beet on 15 September significantly increased polyphenols in leaves in the $1^{\text {st }}$ season as well as significantly reduced fiber in roots in both seasons, compared to that sown one month later. The favorable results of root fiber may be attributed to the suitable weather conditions during growing season.

Table 6: Fiber in roots and poly phenol in leaves as affected by sowing date, methanol and boron foliar application and their interactions in 2014/2015 and 2015/2016 seasons

\begin{tabular}{|c|c|c|c|c|c|c|c|c|c|}
\hline \multirow{2}{*}{\multicolumn{2}{|c|}{ Treatments }} & \multicolumn{8}{|c|}{ Fiber in roots $\%$} \\
\hline & & & 201 & 2015 & & & 2015 & 2016 & \\
\hline \multirow{2}{*}{$\begin{array}{l}\text { Sowing } \\
\text { dates }\end{array}$} & \multirow{2}{*}{$\begin{array}{l}\text { Methanol } \\
\text { levels }\end{array}$} & \multicolumn{8}{|c|}{ Boron levels ( $\mathrm{g}$ boric $\mathrm{acid} / \mathrm{l}$ ) } \\
\hline & & 0 & 0.5 & 1.0 & Mean & 0 & 0.5 & 1.0 & Mean \\
\hline & 0 & 5.46 & 5.42 & 5.08 & 5.32 & 5.48 & 5.11 & 5.07 & 5.22 \\
\hline 15 & $10 \%$ & 5.35 & 5.29 & 4.94 & 5.19 & 5.41 & 5.05 & 5.02 & 5.16 \\
\hline & $20 \%$ & 5.19 & 4.89 & 4.64 & 4.91 & 5.36 & 5.00 & 4.98 & 5.11 \\
\hline \multicolumn{2}{|c|}{ Mean } & 5.33 & 5.20 & 4.89 & 5.14 & 5.41 & 5.05 & 5.02 & 5.16 \\
\hline & 0 & 6.60 & 6.13 & 5.70 & 6.14 & 5.83 & 5.55 & 5.49 & 5.62 \\
\hline & $10 \%$ & 5.95 & 5.88 & 5.43 & 5.76 & 5.57 & 5.33 & 5.31 & 5.40 \\
\hline October & $20 \%$ & 5.67 & 5.64 & 5.29 & 5.53 & 5.39 & 5.26 & 5.23 & 5.29 \\
\hline \multicolumn{2}{|c|}{ Mean } & 6.07 & 5.89 & 5.47 & 5.81 & 5.60 & 5.38 & 5.34 & 5.44 \\
\hline Methanol & 0 & 6.03 & 5.78 & 5.39 & 5.73 & 5.65 & 5.33 & 5.28 & 5.42 \\
\hline$x$ & $10 \%$ & 5.65 & 5.59 & 5.18 & 5.47 & 5.49 & 5.19 & 5.17 & 5.28 \\
\hline Boron & $20 \%$ & 5.43 & 5.27 & 4.97 & 5.22 & 5.37 & 5.13 & 5.10 & 5.20 \\
\hline \multicolumn{2}{|c|}{ Mean } & 5.70 & 5.54 & 5.18 & & 5.51 & 5.22 & 5.18 & \\
\hline \multicolumn{10}{|c|}{ LSD at 0.05 level for: } \\
\hline \multicolumn{2}{|c|}{ Sowing dates $(A)$} & \multicolumn{2}{|c|}{0.06} & $A \times C$ & NS & A & 0.04 & $A \times C$ & 0.07 \\
\hline \multicolumn{2}{|c|}{ Methanol levels (B) } & \multicolumn{2}{|c|}{0.07} & $B \times C$ & NS & $\mathrm{B}$ & 0.05 & $B \times C$ & NS \\
\hline \multicolumn{2}{|c|}{ Boron levels $(\mathrm{C})$} & \multicolumn{2}{|c|}{0.07} & $\mathrm{AxB} \times \mathrm{C}$ & NS & C & 0.05 & $\mathrm{AxB} B \mathrm{C}$ & NS \\
\hline \multirow{2}{*}{\multicolumn{2}{|c|}{$A \times B$}} & \multicolumn{2}{|c|}{0.10} & & & $A \times B$ & 0.07 & & \\
\hline & & \multicolumn{8}{|c|}{ Poly phenol in leaves \% } \\
\hline \multirow{3}{*}{$\begin{array}{c}15^{\text {th }} \\
\text { September }\end{array}$} & 0 & 3.10 & 3.55 & 3.94 & 3.53 & 3.88 & 4.03 & 4.05 & 3.99 \\
\hline & $10 \%$ & 3.48 & 3.70 & 4.05 & 3.74 & 4.37 & 4.55 & 5.15 & 4.69 \\
\hline & $20 \%$ & 3.59 & 3.84 & 4.24 & 3.89 & 4.82 & 5.56 & 5.97 & 5.45 \\
\hline \multicolumn{2}{|c|}{ Mean } & 3.39 & 3.70 & 4.07 & 3.72 & 4.36 & 4.71 & 5.06 & 4.71 \\
\hline & 0 & 2.53 & 2.93 & 3.20 & 2.88 & 3.11 & 3.22 & 3.43 & 3.25 \\
\hline 15 & $10 \%$ & 2.74 & 2.99 & 3.38 & 3.04 & 3.37 & 3.68 & 3.71 & 3.59 \\
\hline October & $20 \%$ & 2.95 & 3.22 & 3.52 & 3.23 & 3.23 & 3.79 & 3.86 & 3.63 \\
\hline \multicolumn{2}{|c|}{ Mean } & 2.74 & 3.04 & 3.36 & 3.05 & 3.24 & 3.56 & 3.67 & 3.49 \\
\hline Methanol & 0 & 2.82 & 3.24 & 3.57 & 3.21 & 3.49 & 3.63 & 3.74 & 3.62 \\
\hline $\mathrm{x}$ & $10 \%$ & 3.11 & 3.35 & 3.71 & 3.39 & 3.87 & 4.12 & 4.43 & 4.14 \\
\hline Boron & $20 \%$ & 3.27 & 3.53 & 3.88 & 3.56 & 4.02 & 4.67 & 4.92 & 4.54 \\
\hline \multicolumn{2}{|c|}{ Mean } & 3.07 & 3.37 & 3.72 & & 3.80 & 4.14 & 4.36 & \\
\hline \multicolumn{10}{|c|}{ LSD at 0.05 level for: } \\
\hline \multicolumn{2}{|c|}{ Sowing dates $(A)$} & \multicolumn{2}{|c|}{0.03} & $A \times C$ & NS & A & NS & $A \times C$ & NS \\
\hline Methanol lev & Is (B) & & & $\mathrm{B} \times \mathrm{C}$ & 0.07 & B & 0.43 & $B \times C$ & NS \\
\hline Boron levels & & & & $\mathrm{AxB} \times \mathrm{C}$ & NS & C & 0.43 & $\mathrm{AxB} \times \mathrm{C}$ & NS \\
\hline$A \times B$ & & & & & & $A \times B$ & NS & & \\
\hline
\end{tabular}


Methanol and boron application significantly affected fiber and polyphenols percentages. The highest doses of methanol and/or boron applications increased polyphenol\% in leaves and decreased fiber\% in roots, in both seasons.

Worthy mention that the recoded fiber\% was generally considered in the normal range of root fiber content, whereas sugar beet root contains about $75 \%$ water, $18 \%$ sugar and approximately $5 \%$ cell walls, the fiber includes three main fractions, pectins, cellulose and arabinose polymers. These findings coincided with those of Chaitanya et al. (2014), who noticed very close correlation among pectin sugars, primary cell walls and boron nutrition. The gradual increase in polyphenol ratio indicates that sugar beet plants were healthy under different concentrations of boron and methanol. These findings could be related to the fundamental role of natural antioxidants in general biological cells. In this respect, Larson (1988) reported that poly phenol is especially common in leaves, which is important in plants for normal growth and defense against infection and injury.

The interaction between sowing date and methanol application had a significant effect on fiber\% in both seasons, while the interaction between sowing date and boron application significantly affected fiber\% in the $2^{\text {nd }}$ season only. Furthermore, the highest values of polyphenol were recorded by the foliar application of $20 \%$ methanol solution and $1 \mathrm{~g}$ boric acid/l in the $1^{\text {st }}$ season compared to the lower application levels.

\section{B. Juice quality and chemical constituents: \\ 1. Sodium, potassium and alpha amino-N concentrations:}

Data in Table 7 showed that sowing date significantly influenced K-content in sugar beet roots, in both seasons as well as $\mathrm{Na}$ and $\alpha$-amino $N$ contents in the $2^{\text {nd }}$ season.
Delaying sowing date exhibited an increase in juice impurities content in both seasons. These results are in line with those obtained by Ismail et al. (2006), who confirmed that delaying sowing date led to increasing impurities content.

Results showed that increasing methanol levels up to $20 \%$ led to significant and gradually reductions in juice impurities, in both seasons. It could be noticed that there are an inverse relationships between methanol concentrations and root contents of impurities.

In the same Table, data showed that values of impurities were significantly reduced with increasing boron levels, in both seasons. These results are in harmony with those obtained by Armin and Asgharipour (2012).

The interaction between sowing dates and methanol applications showed a significant effect on K-content in the $2^{\text {nd }}$ season. The interaction between methanol and boron levels attained significant effects on root $\mathrm{K}$ and $\mathrm{Na}$ contents in both seasons. The interaction between sowing dates and boron levels significantly affected $\mathrm{Na}$, in the $1^{\text {st }}$ season and $\alpha$-amino- $N$, in the $2^{\text {nd }}$ one.

\section{Sucrose and extractable sugar percentages:}

Results in Table 8 manifest that sowing date had a significant effect on sucrose $\%$, in both seasons and extractable sugar\% in the $1^{\text {st }}$ one. Earlier sowing of sugar beet on 15 September led to significant increases in the values of sucrose amounted to 1.17 and $0.67 \%$, compared to delaying sowing date to 15 October, in the $1^{\text {st }}$ and $2^{\text {nd }}$ one, respectively, corresponding to $1.42 \%$ in extractable sugar, in the $1^{\text {st }}$ season. These results are in agreement with those mentioned by Osman et al. (2007) and llkaee et al. (2016). 
Table 7: Sodium, potassium and alpha-amino $\mathrm{N}$ concentrations as affected by sowing date, methanol and boron foliar application and their interactions in 2014/2015 and 2015/2016

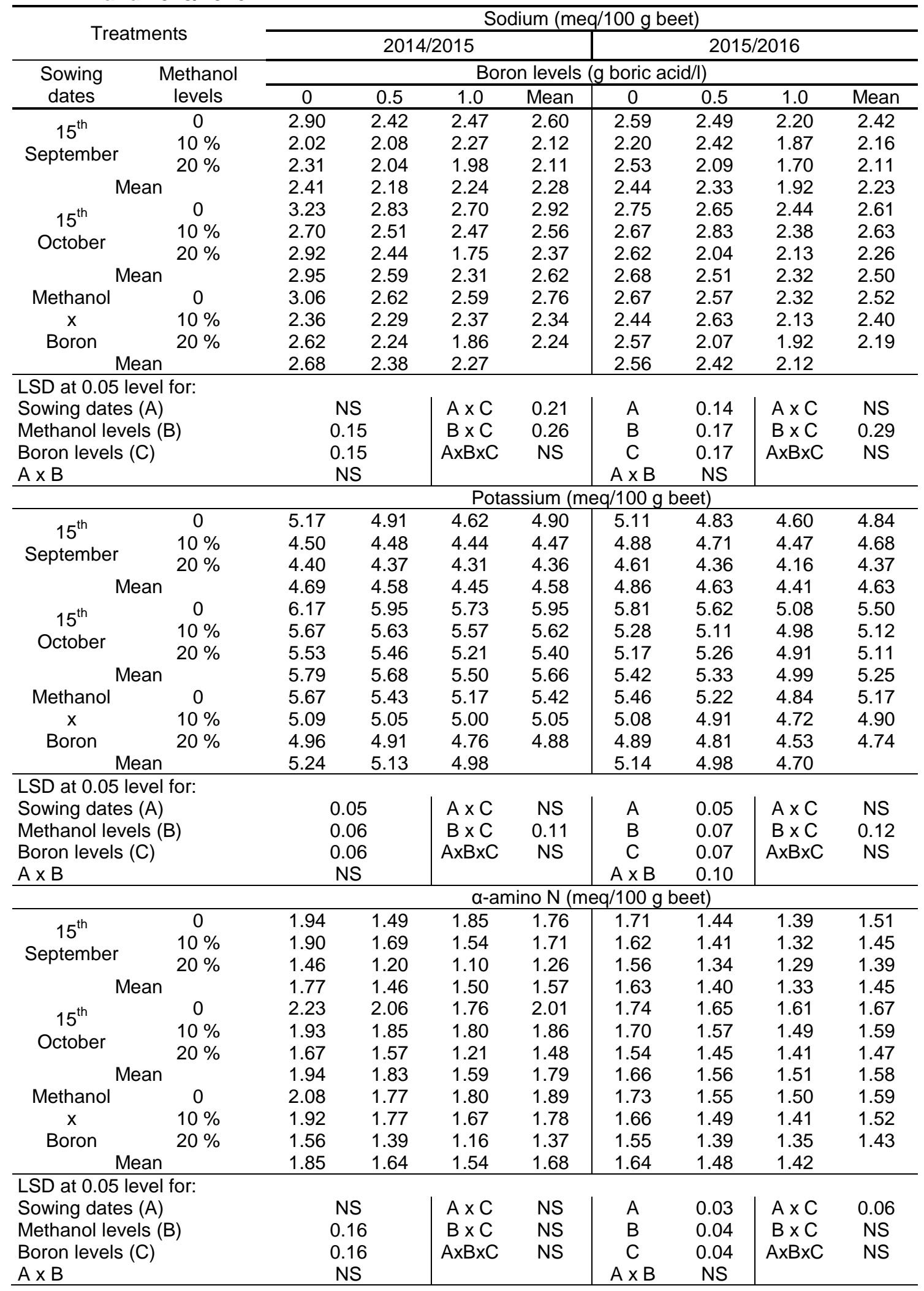


Table 8: Sucrose and extractable sugar percentages as affected by sowing date, methanol and boron foliar application and their interactions in 2014/2015 and 2015/2016 seasons

\begin{tabular}{|c|c|c|c|c|c|c|c|c|c|}
\hline \multirow{2}{*}{\multicolumn{2}{|c|}{ Treatments }} & \multicolumn{8}{|c|}{ Sucrose \% } \\
\hline & & \multicolumn{4}{|c|}{$2014 / 2015$} & \multicolumn{4}{|c|}{$2015 / 2016$} \\
\hline \multirow{2}{*}{$\begin{array}{l}\text { Sowing } \\
\text { dates }\end{array}$} & \multirow{2}{*}{$\begin{array}{l}\text { Methanol } \\
\text { levels }\end{array}$} & \multicolumn{8}{|c|}{ Boron levels (g boric acid/l) } \\
\hline & & 0 & 0.5 & 1.0 & Mean & 0 & 0.5 & 1.0 & Mean \\
\hline \multirow{3}{*}{$\begin{array}{c}15^{\text {th }} \\
\text { September }\end{array}$} & 0 & 18.15 & 18.42 & 19.01 & 18.53 & 17.55 & 18.12 & 18.62 & 18.09 \\
\hline & $10 \%$ & 18.69 & 18.85 & 19.36 & 18.97 & 17.91 & 18.42 & 18.76 & 18.36 \\
\hline & $20 \%$ & 18.95 & 19.21 & 19.95 & 19.37 & 18.77 & 19.67 & 20.83 & 19.75 \\
\hline \multicolumn{2}{|c|}{ Mean } & 18.60 & 18.82 & 19.44 & 18.95 & 18.07 & 18.73 & 19.40 & 18.74 \\
\hline \multirow{3}{*}{$\begin{array}{c}15^{\text {th }} \\
\text { October }\end{array}$} & 0 & 17.01 & 17.12 & 18.04 & 17.39 & 16.69 & 17.40 & 18.04 & 17.38 \\
\hline & $10 \%$ & 17.43 & 17.75 & 18.20 & 17.79 & 17.76 & 18.12 & 18.54 & 18.14 \\
\hline & $20 \%$ & 17.83 & 18.00 & 18.66 & 18.16 & 18.20 & 18.80 & 19.09 & 18.70 \\
\hline \multicolumn{2}{|c|}{ Mean } & 17.42 & 17.62 & 18.30 & 17.78 & 17.55 & 18.11 & 18.56 & 18.07 \\
\hline Methanol & 0 & 17.58 & 17.77 & 18.53 & 17.96 & 17.12 & 17.76 & 18.33 & 17.74 \\
\hline $\mathrm{x}$ & $10 \%$ & 18.06 & 18.30 & 18.78 & 18.38 & 17.83 & 18.27 & 18.65 & 18.25 \\
\hline Boron & $20 \%$ & 18.39 & 18.60 & 19.31 & 18.77 & 18.48 & 19.24 & 19.96 & 19.23 \\
\hline \multicolumn{2}{|c|}{ Mean } & 18.01 & 18.22 & 18.87 & & 17.81 & 18.42 & 18.98 & \\
\hline \multirow{2}{*}{\multicolumn{2}{|c|}{$\begin{array}{l}\text { LSD at } 0.05 \text { level for: } \\
\text { Sowing dates }(A)\end{array}$}} & \multirow{2}{*}{\multicolumn{2}{|c|}{0.24}} & & & & & & \\
\hline & & & & $A \times C$ & NS & $A$ & 0.23 & $A \times C$ & NS \\
\hline \multicolumn{2}{|c|}{ Methanol levels (B) } & \multicolumn{2}{|c|}{0.29} & $B \times C$ & NS & B & 0.28 & $B \times C$ & NS \\
\hline \multicolumn{2}{|c|}{ Boron levels $(\mathrm{C})$} & \multicolumn{2}{|c|}{0.29} & $\mathrm{AxB} \times \mathrm{C}$ & NS & C & 0.28 & $\mathrm{AxB} \times \mathrm{C}$ & NS \\
\hline \multirow{2}{*}{\multicolumn{2}{|c|}{$A \times B$}} & \multicolumn{2}{|c|}{ NS } & & & $A \times B$ & 0.40 & & \\
\hline & & \multicolumn{8}{|c|}{ Extractable sugar \% } \\
\hline \multirow{3}{*}{$\begin{array}{l}15^{\text {th }} \\
\text { September }\end{array}$} & 0 & 15.44 & 15.92 & 16.46 & 15.94 & 14.95 & 15.63 & 16.22 & 15.60 \\
\hline & $10 \%$ & 16.20 & 16.41 & 16.94 & 16.52 & 15.41 & 15.97 & 16.44 & 15.94 \\
\hline & $20 \%$ & 16.55 & 16.91 & 17.70 & 17.05 & 16.28 & 17.33 & 18.59 & 17.40 \\
\hline \multicolumn{2}{|c|}{ Mean } & 16.06 & 16.41 & 17.03 & 16.50 & 15.55 & 16.31 & 17.08 & 16.31 \\
\hline \multirow{3}{*}{$\begin{array}{c}15^{\text {th }} \\
\text { October }\end{array}$} & 0 & 14.03 & 14.28 & 15.32 & 14.55 & 13.96 & 14.73 & 15.48 & 14.72 \\
\hline & $10 \%$ & 14.67 & 15.05 & 15.52 & 15.08 & 15.12 & 15.52 & 16.04 & 15.56 \\
\hline & $20 \%$ & 15.13 & 15.40 & 16.28 & 15.60 & 15.62 & 16.31 & 16.65 & 16.20 \\
\hline \multicolumn{2}{|c|}{ Mean } & 14.61 & 14.91 & 15.71 & 15.08 & 14.90 & 15.52 & 16.06 & 15.49 \\
\hline Methanol & 0 & 14.74 & 15.10 & 15.89 & 15.24 & 14.45 & 15.18 & 15.85 & 15.16 \\
\hline$x$ & $10 \%$ & 15.44 & 15.73 & 16.23 & 15.80 & 15.27 & 15.74 & 16.24 & 15.75 \\
\hline Boron & $20 \%$ & 15.84 & 16.15 & 16.99 & 16.33 & 15.95 & 16.82 & 17.62 & 16.80 \\
\hline & & 15.34 & 15.66 & 16.37 & & 15.22 & 15.92 & 16.57 & \\
\hline LSD at 0.05 & el & & & & & & & & \\
\hline Sowing date & & 0. & & $A \times C$ & NS & $A$ & NS & $A \times C$ & NS \\
\hline Methanol lev & Is (B) & 0. & & $B \times C$ & NS & B & 0.28 & $B \times C$ & NS \\
\hline Boron levels & & 0. & & $A x B \times C$ & NS & $C$ & 0.28 & $A x B x C$ & NS \\
\hline$A \times B$ & & $\mathrm{~N}$ & & & & $A \times B$ & 0.39 & & \\
\hline
\end{tabular}


Data in Table 8 cleared a statistical positive response of sucrose and extractable sugar percentages to the application of methanol in both seasons. Raising concentrations of methanol application to 10 and $20 \%$ caused significant increases in the values of sucrose amounted to 0.42 and $0.81 \%$, corresponding to 0.56 and $1.09 \%$ in the extractable sugar, respectively in the $1^{\text {st }}$ season, as well as 0.51 and $1.49 \%$ in sucrose, corresponding to 0.59 and $1.64 \%$ in extractable sugar, respectively in the $2^{\text {nd }}$ season, compared to the check treatment. These results are in agreement with those obtained by Abido (2012). In addition, Zbieć et al. (2003) and Nadali et al. (2010) explained that the leaves of many plants have covered by methylobacterium bacteria, which are capable to grow on methanol and generate doubling of $\mathrm{CO}_{2}$ content, which lead to two folds the sucrose to be produced through Calvin cycle from the two source of $\mathrm{CO}_{2}$.

Data in the same Table cleared that sucrose and extractable sugar percentages were significantly affected by the applied boron levels. Raising concentrations of boron to 0.5 and $1.0 \mathrm{~g}$ boric acid// increased the values of sucrose by 0.21 and $0.86 \%$, corresponding to 0.32 and $1.03 \%$ in the extracted sugar, respectively in the $1^{\text {st }}$ season, as well as 0.61 and $1.17 \%$ in sucrose, corresponding to 0.70 and $1.35 \%$ in the extracted sugar, respectively in the $2^{\text {nd }}$ one, compared to the check treatment. These results are in harmony with those mentioned by Armin and Asgharipour (2012). These results assured the importance of boron element in metabolic translocation process.

All studied interactions showed insignificant effects on sucrose and extractable sugar percentages in both seasons, except that between sowing dates and methanol levels, which had a significant influence on these traits, in the $2^{\text {nd }}$ one. Sowing sugar beet on 15 September achieved the highest values of sucrose and extractable sugar percentages, when plants were sprayed with $20 \%$ methanol solution in the $2^{\text {nd }}$ season.

\section{Juice purity and sugar lost to molasses percentages:}

Data in Table 9 revealed that purity and sugar lost to molasses (SLM) percentages were significantly affected by sowing date. Earlier sowing of sugar beet on 15 September increased the values of purity\% by 1.73 and $1.03 \%$, in the $1^{\text {st }}$ and $2^{\text {nd }}$ season, respectively, compared to that sown one month later. These results are in agreement with those found by Osman et al. (2007) and Mosa (2009). On the contrary, sowing sugar beet earlier appreciably decreased the percentage of sugar lost to molasses by 0.25 and $0.16 \%$, in the $1^{\text {st }}$ and $2^{\text {nd }}$ season, successively. The better quality characteristics of beets sown earlier, in respect to these two traits, is probably attributed to favorable conditions for beet plants, especially lower night temperature degrees during ripening stage before harvesting (Table 2), which ensured lower contents of impurities (Table 7) and higher sucrose\% (Table 8).

Results showed that increasing methanol levels up to $20 \%$ led to significant and gradual increases in purity and sugar lost to molasses percentages, in both seasons. These findings are in agreement with those mentioned by Abido (2012).

Supplying sugar beet plants with boron resulted in a significant and an increase in purity and a decrease in sugar lost to molasses.

The interaction between sowing date and boron levels significantly affected juice purity and sugar lost to molasses percentages in the $1^{\text {st }}$ season. Concerning the interaction between methanol and boron levels, the highest value of purity\% was obtained from beets sprayed with a $20 \%$ methanol solution and $1 \mathrm{~g}$ boric acid/l in the $1^{\text {st }}$ season. 
Table 9: Purity and sugar lost to molasses percentages as affected by sowing date, methanol and boron foliar application and their interactions in 2014/2015 and 2015/2016 seasons

\begin{tabular}{|c|c|c|c|c|c|c|c|c|c|}
\hline \multirow{2}{*}{\multicolumn{2}{|c|}{ Treatments }} & \multicolumn{8}{|c|}{ Purity \% } \\
\hline & & \multicolumn{4}{|c|}{$2014 / 2015$} & \multicolumn{4}{|c|}{$2015 / 2016$} \\
\hline \multirow{2}{*}{$\begin{array}{l}\text { Sowing } \\
\text { dates }\end{array}$} & \multirow{2}{*}{$\begin{array}{c}\text { Methanol } \\
\text { levels }\end{array}$} & \multicolumn{8}{|c|}{ Boron levels (g boric acid/l) } \\
\hline & & 0 & 0.5 & 1.0 & Mean & 0 & 0.5 & 1.0 & Mean \\
\hline \multirow{3}{*}{$\begin{array}{c}15^{\text {th }} \\
\text { September }\end{array}$} & 0 & 91.50 & 92.52 & 92.65 & 92.22 & 91.71 & 92.47 & 93.08 & 92.42 \\
\hline & $10 \%$ & 92.92 & 93.11 & 93.27 & 93.10 & 92.43 & 92.74 & 93.53 & 92.90 \\
\hline & $20 \%$ & 93.20 & 93.69 & 94.07 & 93.65 & 92.75 & 93.72 & 94.46 & 93.64 \\
\hline \multicolumn{2}{|c|}{ Mean } & 92.54 & 93.11 & 93.33 & 92.99 & 92.30 & 92.97 & 93.69 & 92.99 \\
\hline \multirow{3}{*}{$\begin{array}{c}15^{\text {th }} \\
\text { October }\end{array}$} & 0 & 89.61 & 90.34 & 91.30 & 90.42 & 90.49 & 91.22 & 92.13 & 91.28 \\
\hline & $10 \%$ & 90.93 & 91.33 & 91.64 & 91.30 & 91.60 & 91.86 & 92.54 & 92.00 \\
\hline & $20 \%$ & 91.26 & 91.85 & 93.07 & 92.06 & 92.04 & 92.71 & 93.04 & 92.60 \\
\hline \multicolumn{2}{|c|}{ Mean } & 90.60 & 91.17 & 92.00 & 91.26 & 91.38 & 91.93 & 92.57 & 91.96 \\
\hline Methanol & 0 & 90.55 & 91.43 & 91.98 & 91.32 & 91.10 & 91.84 & 92.61 & 91.85 \\
\hline$x$ & $10 \%$ & 91.93 & 92.22 & 92.46 & 92.20 & 92.02 & 92.30 & 93.04 & 92.45 \\
\hline Boron & $20 \%$ & 92.23 & 92.77 & 93.57 & 92.86 & 92.40 & 93.22 & 93.75 & 93.12 \\
\hline \multicolumn{2}{|c|}{ Mean } & 91.57 & 92.14 & 92.67 & & 91.84 & 92.45 & 93.13 & \\
\hline \multicolumn{10}{|c|}{ LSD at 0.05 level for: } \\
\hline \multicolumn{2}{|c|}{ Sowing dates (A) } & \multicolumn{2}{|c|}{0.19} & $A \times C$ & 0.33 & $A$ & 0.14 & $A \times C$ & NS \\
\hline \multicolumn{2}{|c|}{ Methanol levels (B) } & \multicolumn{2}{|c|}{0.24} & $B \times C$ & 0.41 & B & 0.17 & $B \times C$ & NS \\
\hline \multicolumn{2}{|c|}{ Boron levels (C) } & \multicolumn{2}{|c|}{0.24} & $\mathrm{AxB} \times \mathrm{C}$ & NS & C & 0.17 & $\mathrm{~A} \times \mathrm{B} \times \mathrm{C}$ & NS \\
\hline \multirow{2}{*}{\multicolumn{2}{|c|}{$A \times B$}} & \multicolumn{2}{|c|}{ NS } & & & $A \times B$ & NS & & \\
\hline & & \multicolumn{8}{|c|}{ Sugar lost to molasses \% } \\
\hline \multirow{3}{*}{$\begin{array}{c}15^{\text {th }} \\
\text { September }\end{array}$} & 0 & 2.11 & 1.90 & 1.96 & 1.99 & 2.00 & 1.88 & 1.80 & 1.89 \\
\hline & $10 \%$ & 1.89 & 1.84 & 1.82 & 1.85 & 1.90 & 1.85 & 1.72 & 1.82 \\
\hline & $20 \%$ & 1.80 & 1.70 & 1.66 & 1.72 & 1.89 & 1.74 & 1.64 & 1.76 \\
\hline \multicolumn{2}{|c|}{ Mean } & 1.94 & 1.81 & 1.81 & 1.85 & 1.93 & 1.82 & 1.72 & 1.82 \\
\hline \multirow{3}{*}{$\begin{array}{c}15^{\text {th }} \\
\text { October }\end{array}$} & 0 & 2.37 & 2.24 & 2.12 & 2.25 & 2.13 & 2.07 & 1.95 & 2.05 \\
\hline & $10 \%$ & 2.15 & 2.10 & 2.08 & 2.11 & 2.04 & 2.00 & 1.90 & 1.98 \\
\hline & $20 \%$ & 2.10 & 2.00 & 1.78 & 1.96 & 1.98 & 1.89 & 1.84 & 1.90 \\
\hline & & 2.21 & 2.11 & 1.99 & 2.10 & 2.05 & 1.99 & 1.90 & 1.98 \\
\hline Methanol & 0 & 2.24 & 2.07 & 2.04 & 2.12 & 2.07 & 1.98 & 1.88 & 1.97 \\
\hline$x$ & $10 \%$ & 2.02 & 1.97 & 1.95 & 1.98 & 1.97 & 1.93 & 1.81 & 1.90 \\
\hline Boron & $20 \%$ & 1.95 & 1.85 & 1.72 & 1.84 & 1.93 & 1.81 & 1.74 & 1.83 \\
\hline & & 2.07 & 1.96 & 1.90 & & 1.99 & 1.91 & 1.81 & \\
\hline LSD at 0.05 & evel for: & & & & & & & & \\
\hline Sowing date & & & & $A \times C$ & 0.07 & A & 0.02 & $A \times C$ & NS \\
\hline Methanol lev & Is (B) & & & $B \times C$ & NS & B & 0.02 & $B \times C$ & NS \\
\hline Boron levels & & & & $A \times B \times C$ & NS & $\mathrm{C}$ & 0.02 & $\mathrm{AxB} \times \mathrm{C}$ & NS \\
\hline$A \times B$ & & & & & & $A \times B$ & NS & & \\
\hline
\end{tabular}




\section{Top, root and sugar yields/fed:}

Data in Table 10 demonstrated that sugar beet sown on 15 September significantly recorded higher top, root and sugar yields/fed than that planted on the $15^{\text {th }}$ of October, in both seasons. The relative advantage of early sowing may be due to the appropriate meteorological factors not only for a rapid growth, but also for sugar storage by the end of the season, which positively resulted in higher values of root length, root diameter and root fresh weight/plant (Table 3), LAl and NAR (Table 4), photosynthetic pigments (Table 5), lower contents of impurities in roots (Table 7), higher sucrose and extractable sugar percentages (Table 8) and ultimately participated in getting higher yields of tops, roots and sugar/fed, compared to late sowing. Likewise, Mosa (2009) mentioned that earlier sowing of sugar beet improved the recorded values of individual plants, which in turn affected the final crop at harvest in terms of top, root and sugar yields. Results cleared that sowing sugar beet on 15 September attained additional increases amounted to 0.93 and 0.64 ton/fed in top yield/fed, corresponding to 0.59 and 2.34 tons/fed in root yield/fed and 0.44 and 0.57 ton/fed in sugar yield, in the $1^{\text {st }}$ and $2^{\text {nd }}$ season, respectively, compared to that sown on 15 October.

The results in Table 10 revealed that the applied concentrations of methanol and/or boron increased top, root and sugar yields/fed appreciably in both seasons. These findings are in line with these obtained by Nadali et al. (2010), Abido (2012) and El-Geddawy and Makhlouf (2015). Raising methanol levels to 10 and $20 \%$ led to gradual increases in root fresh yield amounted to 0.44 and 0.95 ton/fed in the $1^{\text {st }}$ season, corresponding to 0.71 and
2.45 tons/fed in the $2^{\text {nd }}$ one, respectively. Meantime, the increases in sugar yield amounted to 0.20 and 0.42 ton/fed in the $1^{\text {st }}$ season, corresponding to 0.23 and 0.78 ton/fed in the $2^{\text {nd }}$ one, successively, compared to the check treatment. In sugar beet, white sugar yield is a component of accumulated dry weight of the roots, and the maximum white sugar yield is obtained when dry weight of the roots is in its highest amount (Ranji et al., 2000). Therefore, it is possible to improve white sugar yield by increasing root yield through foliar application of methanol.

Increasing boron levels to 0.5 and $1.0 \mathrm{~g}$ boric acid/l gave increments in root yield amounted to 0.39 and 0.80 ton/fed in the $1^{\text {st }}$ season, corresponding to 0.38 and 0.74 ton/fed in the $2^{\text {nd }}$ one, respectively. Moreover, the increments in sugar yield amounted to 0.14 and 0.38 ton/fed in the $1^{\text {st }}$ season, corresponding to 0.22 and 0.43 ton/fed in the $2^{\text {nd }}$ one, successively. In this respect, Hellal et al. (2009) showed that the application of boron significantly encouraged the balance of nutrients, thus getting higher yield in the prevailing conditions.

The interaction between sowing dates and foliar spraying of methanol showed significant influences on top and root yields in both seasons as well as sugar yield in the $2^{\text {nd }}$ one. Sowing sugar beet on 15 September achieved the highest averages of top, root and sugar yields/fed compared to sowing on 15 October, when plants were sprayed with $20 \%$ methanol solution in both seasons. The interaction between sowing dates and boron foliar application had a significant influence on root yield/fed in the $1^{\text {st }}$ season and top yield in the $2^{\text {nd }}$ one, while the same interaction had an insignificant on sugar yield/fed. 
Table 10: Top, root and sugar yields as affected by sowing date, methanol and boron foliar application and their interactions in 2014/2015 and 2015/2016 seasons.

\begin{tabular}{|c|c|c|c|c|c|c|c|c|c|}
\hline \multirow{2}{*}{\multicolumn{2}{|c|}{ Treatments }} & \multicolumn{8}{|c|}{ Top yield (ton/fed) } \\
\hline & & \multicolumn{4}{|c|}{$2014 / 2015$} & \multicolumn{4}{|c|}{$2015 / 2016$} \\
\hline \multirow{2}{*}{$\begin{array}{c}\text { Sowing } \\
\text { dates }\end{array}$} & \multirow{2}{*}{$\begin{array}{l}\text { Methanol } \\
\text { levels }\end{array}$} & \multicolumn{8}{|c|}{ Boron levels ( $\mathrm{g}$ boric acid/l) } \\
\hline & & 0 & 0.5 & 1.0 & Mean & 0 & 0.5 & 1.0 & Mean \\
\hline & 0 & 10.19 & 10.60 & 10.87 & 10.55 & 9.96 & 10.33 & 10.54 & 10.28 \\
\hline $15^{110}$ & $10 \%$ & 10.96 & 11.09 & 11.33 & 11.13 & 10.49 & 11.07 & 11.23 & 10.93 \\
\hline September & $20 \%$ & 11.93 & 11.97 & 12.07 & 11.99 & 10.78 & 11.19 & 11.26 & 11.08 \\
\hline \multicolumn{2}{|c|}{ Mean } & 11.03 & 11.22 & 11.42 & 11.22 & 10.41 & 10.87 & 11.01 & 10.76 \\
\hline $15^{\text {th }}$ & 0 & 10.15 & 10.17 & 10.13 & 10.15 & 9.32 & 10.06 & 10.30 & 9.89 \\
\hline 15 & $10 \%$ & 10.12 & 10.36 & 10.46 & 10.31 & 9.71 & 10.16 & 10.57 & 10.14 \\
\hline & $20 \%$ & 10.25 & 10.38 & 10.58 & 10.40 & 9.74 & 10.34 & 10.91 & 10.33 \\
\hline \multicolumn{2}{|c|}{ Mean } & 10.17 & 10.30 & 10.39 & 10.29 & 9.59 & 10.19 & 10.59 & 10.12 \\
\hline Methanol & 0 & 10.17 & 10.38 & 10.50 & 10.35 & 9.64 & 10.20 & 10.42 & 10.09 \\
\hline $\mathrm{x}$ & $10 \%$ & 10.54 & 10.72 & 10.89 & 10.72 & 10.10 & 10.62 & 10.90 & 10.54 \\
\hline Boron & $20 \%$ & 11.09 & 11.17 & 11.33 & 11.20 & 10.26 & 10.77 & 11.09 & 10.70 \\
\hline \multicolumn{2}{|c|}{ Mean } & 10.60 & 10.76 & 10.91 & & 10.00 & 10.53 & 10.80 & \\
\hline \multirow{6}{*}{\multicolumn{2}{|c|}{$\begin{array}{l}\text { LSD at } 0.05 \text { level for: } \\
\text { Sowing dates }(A) \\
\text { Methanol levels }(B) \\
\text { Boron levels }(C) \\
A \times B\end{array}$}} & & & & & \\
\hline & & \multicolumn{2}{|c|}{0.18} & $A \times C$ & NS & A & 0.12 & $A \times C$ & 0.21 \\
\hline & & \multicolumn{2}{|c|}{0.22} & $B \times C$ & NS & B & 0.15 & $B \times C$ & NS \\
\hline & & \multirow{2}{*}{\multicolumn{2}{|c|}{$\begin{array}{l}0.22 \\
0.31\end{array}$}} & $\mathrm{AxB} \times \mathrm{C}$ & NS & C & 0.15 & $\mathrm{AxB} \times \mathrm{C}$ & NS \\
\hline & & & & & & $A \times B$ & 0.21 & & \\
\hline & & & & & Root yie & (ton/fec & & & \\
\hline & 0 & 23.58 & 24.07 & 24.46 & 24.04 & 23.09 & 23.60 & 23.99 & 23.56 \\
\hline Sentember & $10 \%$ & 23.96 & 24.34 & 24.95 & 24.42 & 23.55 & 23.78 & 23.98 & 23.77 \\
\hline September & $20 \%$ & 24.80 & 25.18 & 25.66 & 25.21 & 25.65 & 26.27 & 26.56 & 26.16 \\
\hline \multicolumn{2}{|c|}{ Mean } & 24.11 & 24.53 & 25.03 & 24.56 & 24.10 & 24.55 & 24.84 & 24.50 \\
\hline & 0 & 23.21 & 23.58 & 23.91 & 23.57 & 20.84 & 21.02 & 21.11 & 20.99 \\
\hline 15 & $10 \%$ & 23.68 & 24.02 & 24.48 & 24.06 & 21.65 & 22.09 & 22.86 & 22.20 \\
\hline uctober & $20 \%$ & 23.98 & 24.36 & 24.52 & 24.29 & 22.95 & 23.26 & 23.68 & 23.30 \\
\hline & & 23.62 & 23.99 & 24.31 & 23.97 & 21.81 & 22.12 & 22.55 & 22.16 \\
\hline Methanol & 0 & 23.39 & 23.83 & 24.19 & 23.80 & 21.97 & 22.31 & 22.55 & 22.28 \\
\hline $\mathrm{x}$ & $10 \%$ & 23.82 & 24.18 & 24.72 & 24.24 & 22.60 & 22.94 & 23.42 & 22.99 \\
\hline Boron & $20 \%$ & 24.39 & 24.77 & 25.09 & 24.75 & 24.30 & 24.77 & 25.12 & 24.73 \\
\hline & & 23.87 & 24.26 & 24.67 & & 22.96 & 23.34 & 23.70 & \\
\hline LSD at 0.05 & & & & & & & & & \\
\hline Sowing date & & & & $A \times C$ & 0.14 & & 0.28 & $A \times C$ & NS \\
\hline Methanol lev & & & & $B \times C$ & NS & B & 0.34 & $B \times C$ & NS \\
\hline Boron levels & & & & $\mathrm{A} \times \mathrm{B} \times \mathrm{C}$ & NS & C & 0.34 & $\mathrm{AxB} \times \mathrm{C}$ & NS \\
\hline$A \times B$ & & & & & & $A \times B$ & 0.48 & & \\
\hline & & & & & ugar yi & (ton/fe & & & \\
\hline & 0 & 3.64 & 3.83 & 4.03 & 3.83 & 3.45 & 3.69 & 3.89 & 3.68 \\
\hline $15^{1 \mathrm{~m}}$ & $10 \%$ & 3.88 & 3.99 & 4.23 & 4.03 & 3.63 & 3.80 & 3.94 & 3.79 \\
\hline September & $20 \%$ & 4.10 & 4.26 & 4.54 & 4.30 & 4.18 & 4.55 & 4.94 & 4.56 \\
\hline & & 3.87 & 4.03 & 4.27 & 4.06 & 3.75 & 4.01 & 4.26 & 4.01 \\
\hline & 0 & 3.26 & 3.37 & 3.67 & 3.43 & 2.92 & 3.09 & 3.27 & 3.09 \\
\hline 15 & $10 \%$ & 3.47 & 3.61 & 3.80 & 3.63 & 3.28 & 3.43 & 3.66 & 3.46 \\
\hline October & $20 \%$ & 3.63 & 3.75 & 3.99 & 3.79 & 3.59 & 3.79 & 3.94 & 3.77 \\
\hline & & 3.45 & 3.58 & 3.82 & 3.62 & 3.26 & 3.44 & 3.63 & 3.44 \\
\hline Methanol & 0 & 3.45 & 3.60 & 3.85 & 3.63 & 3.19 & 3.39 & 3.58 & 3.39 \\
\hline $\mathrm{x}$ & $10 \%$ & 3.68 & 3.80 & 4.01 & 3.83 & 3.45 & 3.61 & 3.80 & 3.62 \\
\hline Boron & $20 \%$ & 3.87 & 4.00 & 4.27 & 4.05 & 3.88 & 4.17 & 4.44 & 4.17 \\
\hline & & 3.66 & 3.80 & 4.04 & & 3.51 & 3.73 & 3.94 & \\
\hline LSD at 0.05 & el for: & & & & & & & & \\
\hline Sowing date & & & & $A \times C$ & NS & A & 0.07 & $A \times C$ & \\
\hline Methanol lev & (B) & & & $B \times C$ & NS & B & 0.09 & $B \times C$ & NS \\
\hline Boron levels & & & & $\mathrm{A} \times \mathrm{B} \times \mathrm{C}$ & NS & C & 0.09 & $\mathrm{~A} \times \mathrm{B} \times \mathrm{C}$ & NS \\
\hline$A \times B$ & & & & & & $A \times B$ & 0.12 & & \\
\hline
\end{tabular}




\section{CONCLUSION}

Under conditions of the present work, it was found that sowing sugar beet earlier on 15 September, sprayed with a solution of 20 $\%$ methanol and $1.0 \mathrm{~g} / \mathrm{l}$ boric acid can be recommended to get the highest root and sugar yields/fed.

\section{REFERENCES}

Abd El-Maged, A.A., B.M. Abou El-Magd and H. G. Abou El-Fotoh (2004). Using some organic compounds for improving sugar beet productivity. J. Agric. Sci., 29(3): 1551-1561, Mansoura Univ., Egypt.

Abido, W.A.E. (2012). Sugar beet productivity as affected by foliar spraying with methanol and boron. Int. J. Agric. Sci., 4(7): 287-292.

Armin, M. and M. Asgharipour (2012). Effect of time and concentration of boron foliar application on yield and quality of sugar beet. Am-Eu. J. Agric. \& Environ. Sci., 12 (4): 444-448.

Association of Official Agricultural Chemist (2005). Association of Official Analytical Chemists. Official methods of analysis, $26^{\text {th }}$ Ed. A.O.A.C., International, Washington, D.C; USA.

Benson, A.A. and A.M. Nonomura (1992). The path of carbon in photosynthesis: methanol inhibition of glycolic acid accumulation. Photosyn. Res. 34:196. (abst. P-522) Presented at the IX ${ }^{\text {th }}$ Int. Cong. Photosyn., Nagoya, Japan. Aug. 30 to Sep. 4.

Blevins, Dale G. and Krystyna M. Lukaszewski (1998). Boron in plant structure and function. Ann. Rev. Plant Physiol. Plant Mol. Biol,.49:481-500.

Chapman, H.D. and R.E. Pratt (1961). Methods of Analysis for Soil, Plants and Water. Dep. Soil, Plant Nutrition, Univ. California, USA.

Deviller, P. (1988). Prevision du sucre melasse sucrerie feanases.129:190-200. [C.F. Cooke, D.A. and R.K. Scott (1993) The Sugar Beet Crop Book].
Dexter, S.T., M.G. Frankes and F.W. Snyder (1967). A rapid and practical method of determining extractable white sugar as may be applied to the evaluation of agronomic practices and grower deliveries in the sugar beet industry. J. Am., Soc., Sugar Beet Technol., 14:433454.

Dordas, C., G.E. Apostolides and O. Goundra (2007). Boron application affects seed yield and seed quality of sugar beets. J. Agric. Sci., 145, 377-384.

El-Geddawy, Dalia I.H. and B.S.I. Makhlouf (2015). Effect of hill spacing and nitrogen and boron fertilization levels on yield and quality attributes in sugar beet. Minufiya J. Agric. Res., 4(1): 959980.

Gobarah, Mirvat and B.B. Mekki (2005). Influence of boron application on yield and juice quality of some sugar beet cultivars grown under saline soil conditions. J. Appl. Sci. Res., 1 (5): 373379.

Hellal, F.A., A.S. Taalab and A.M. Safaa (2009). Influence of nitrogen and boron nutrition on nutrient balance and sugar beet yield grown in calcareous soil. Ozean J. Appl. Sci., 2(1):1-10.

Hemayati, S.S., M.H. Shirzadi, M. Aghaeezadeh, D.F. Taleghani, M.A. Javaheri and A. Aliasghari (2012). Evaluation of sowing and harvesting date effects on yield and quality of five sugar beet cultivars in Jiroft region (autumn planting). J. Sugar Beet, 28(1):13-21.

Ilkaee1, M.N., B. Zohre, B. Amirsaleh and G. Farid (2016). Effect of different planting dates and defoliation on the properties of sugar beet (Beta vulgaris L.). J. Exp. Biol. Agric. Sci., Feb., 4(1).

Ismail, A.M.A., A.H.S. Al-Labbody and N.M.S. Shalaby (2006). Variability and traits relationships in nine sugar beet varieties under three sowing dates. Egypt. J. Plant Breed, 10 (1): 387-406.

Jackson, M.L. (1967). Soil chemical analysis, prentice- hall India private limited, New York. 
Khazaei, S., T.D. Fathollah and D.H. Hassanpour (2015). The Effect of methanol foliarapplication on the tolerance of sugar beet cultivars to drought stress. Biol. Forum - Int. J. 7(1): 1502-1507.

Larson, R.A. (1988). The antioxidants of higher plants. Phytochemistry, 27, 969978.

Le Docte, A. (1927). Commercial determination of sugar beet root using the Sacks Le-Docte Process. Int. Sugar J., 29: 488-492.

Leilah, A.A., M.A. Badawi, E.M. Said, M.H. Ghonema and M.A.E. Abdou (2005). Effect of planting dates, plant population and nitrogen fertilization on sugar beet productivity under the newly reclaimed sandy soli in Egypt. Sci. J. King Univ., 6(1): 95-109.

Markus, D.K., J.P. Mckinnon and A.F. Buccafuri (1982). Automated Analysis of Nitrite and ammonium Nitrogen in soils. New Jersey Agric. Exp. Stn. Publ. No. D15117-84, USA.

Mohammad, A. and R.A. Mohammad (2011). Effect of time and concentration of boron foliar application on yield and quality of sugar beet. Asian J. Plant Sci., 10: 307-311.

Mosa, D.T.M.A. (2009). Effect of sowing and harvesting dates on yield and quality of some sugar beet varieties. M.Sc. Thesis, Fac. Agric., Cairo Univ. Egypt.

Nable, R.O., G.S. Bañuelos and J.G. Paull (1997). Boron toxicity Plant and Soil 193, 181-198.

Nadali, I., F. Paknejad, F. Moradi, S. Vazan, M. Tookalo, Jami AL-Ahmed and M. Pazoki A. (2010). Effect of foliar application of methanol on sugar beet (Beta Vulgaris, L). Australian J. Crop Sci., 4(6): 398-401.

Nonomura, A.M. and A. Benson (1992). The path of carbon in photosynthesis: improved crop yields with methanol. Proc. Natl. Acad. Sci., USA 89:97949798.
Osman, M.S., I.H. EL-Geddawy, M.G. AbdEl-Fadeil and S.A.A.M. Enan (2007). Transplanting using paper pots technique and micro nutrition with relation to juice quality and chemical constituents of sugar beet at different planting dates. Egypt, J. Agric. Res., 85(1): 211-229.

Piper, C.S. (1950). Soil and Plant Anlysis. Inter. Sci. Publ. Inc., New York.

Radford's, P.J. (1967). Growth analysis formulae, their use and abuse. Crop Sci., 7: 171-175.

Ranji, Z., M. Chegini, Gh. Tohidloo and M. Abdollahian-Noghabi (2000). Investigation of drought tolerance on physiological traits in sugar beet related to nitrogen and potassium. (Iranian Sugar beet Res. Inst.) Reports in part of Breeding Res. 64p.

Singleton, V.L., R. Orthofer and R.M. Lamuela-Raventos (1999). Analysis of total phenols and other oxidation substrates and antioxidants by means of Folin-Ciocalteu reagent.Methods Enzymol. 299, 152-178.

Snedecor, G.W. and W.G. Cochran (1981). Statistical methods $7^{\text {th }}$ Ed lowa State Univ. Press, Ames, lowa, USA.

Soltanpour, P. N. (1991). Determination of nutrient availability element toxicity by AB-DTPA. Soil Test and ICPS Adv. Soil Sci., 16:165-190.

Waller, R.A. and D.B. Duncan (1969). A bays rule for symmetric multiple comparison problem. Amer. Stat. Assoc. J., 1485-1503.

Watson, D.J. (1958). The dependence of net assimilation rate on leaf area index. Ann. Bot. Lond. N.S., 22:37-54.

Wettestien, D.V. (1957). Chlorophyll, lethal und submikroskopische formel wechsel der plastiden Exptl .Cell Res. 12: 427433.

Zbieć, I., S. Karczmarczyk and C. Podsiadlo (2003). Response of some cultivated plants to methanol as compared to supplemental irrigation. E. J. Polish Agric. Univ., Agron., 6, 1-7. 


\title{
تحسين حاصل وجودة بنجر السُكَّر المنزرع فى ميعادين بإضافة الميثانول والبورون
}

\author{
باسم صبحي إبراهيم مخلوف(1) ، سـها روضان خليل(2)

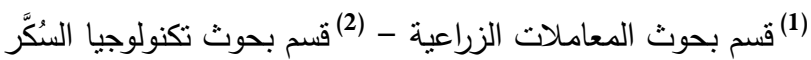 \\ معهد بحوث المحاصيل السُكَّرية - مركز البحوث الزراعية - الجيزة - مصر لئر
}

المُلأَّص العربى

أقيمت تجربتان حقلينان في سنورس - محافظة الفيوم في موسمي 2015/2014 و 2016/2015 لدراسة تأثير ميعادين للزراعة (15 سبتمبر و 15 أكتوبر) والرش الورقي بكل من الميثانول والبورون علي حاصل وجودة بنجر السُكَّر . انتنمل كل ناير

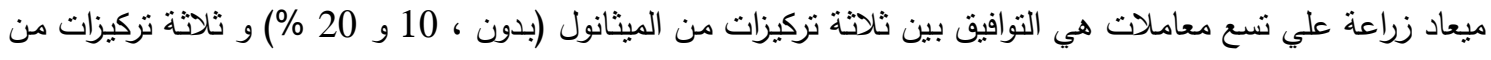
البورون (بدون ، 0.5 و 1.0 جم حمض بوريك/لتز) ). تم توزيع المعاملات في تصميم القطاعات كاملة فئة العشوائية في ثناث

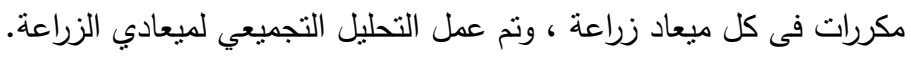

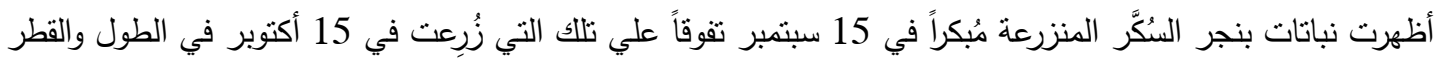

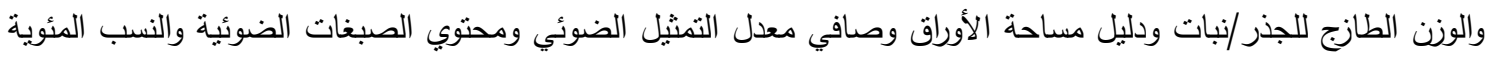

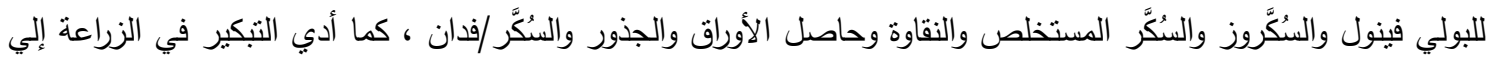

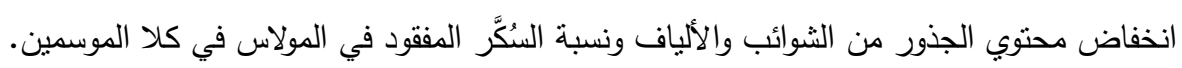

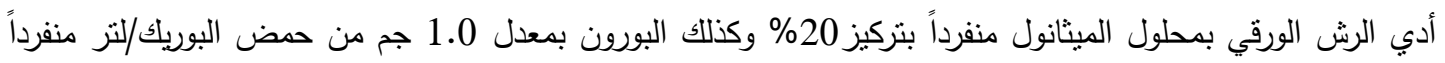

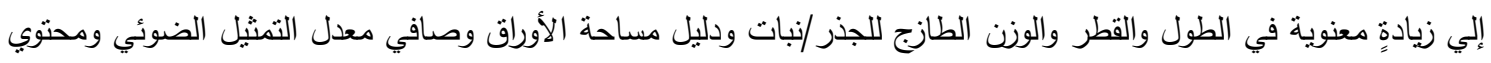

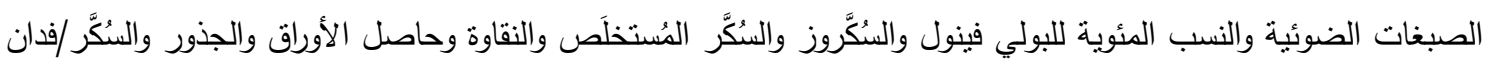
، وإنخفاضٍ في محتوي الجذور من الثوائب والألياف ونسبة السُكَّر المفقود في المولاس في كلا لألا الموسمين.

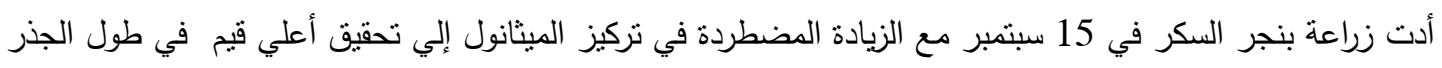

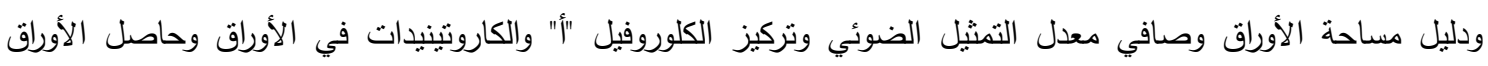

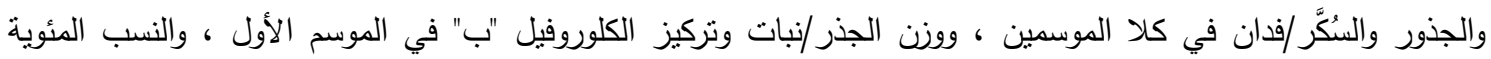

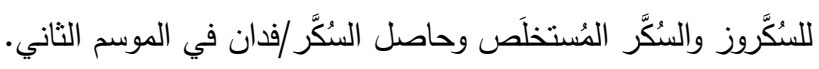

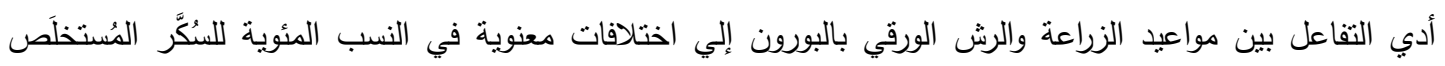

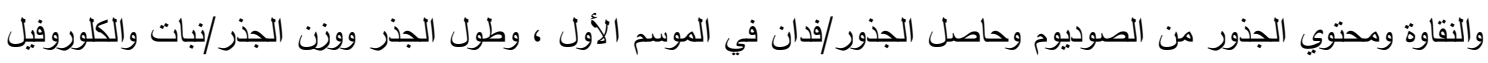

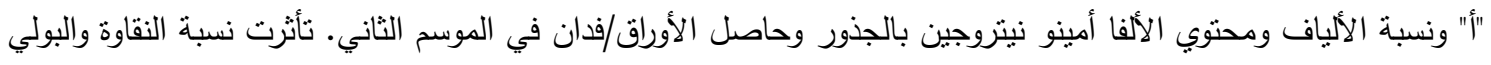

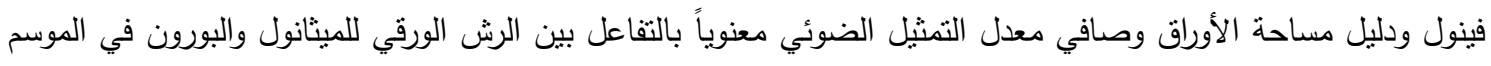

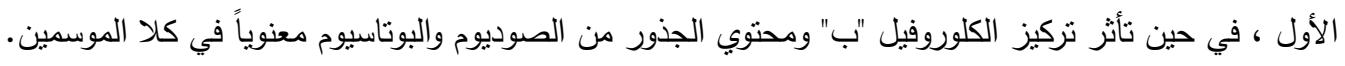

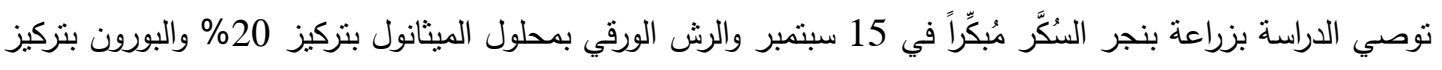

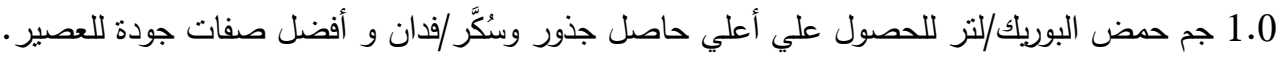


Improvement of yield and quality of sugar beet sown at two dates .............. 